\title{
ON A TRIVIAL ZERO PROBLEM
}

\section{SHAOWEI ZHANG}

\author{
Received 25 October 2002
}

One trivial zero phenomenon for $p$-adic analytic function is considered. We then prove that the first derivative of this function is essentially the Kummer class associated with $p$.

2000 Mathematics Subject Classification: 11S40.

1. Introduction. In this paper we always fix an odd prime $p>2$. For $n \geq 1$, fix a $p^{n}$ th primitive root of unity $\zeta_{p^{n}}$ such that $\zeta_{p^{n+1}}^{p}=\zeta_{p^{n}}$. Let $K_{n}=\mathbb{Q}_{p}\left(\zeta_{p^{n}}\right)$ and $u=$ $\underline{\lim } O_{K_{n}}^{\times}$. For $\beta \in \mathcal{U}$, we will define a 1-admissible distribution $\mu_{\beta} \in \mathscr{D}_{1}\left(\mathbb{Q}_{p}, \mathbb{Q}_{p}[-1]\right)^{\Phi=1}$ (see Section 3). Consider the integral

$$
\psi_{k}(\beta)=\int_{\mathbb{Z}_{p}^{\times}} x^{k} \mu_{\beta},
$$

then we have $\psi_{k}(\beta)=\left(1-p^{k-1}\right) \cdot \int_{\mathbb{Z}_{p}} x^{k} \mu_{\beta}$, so it will have a trivial zero at $k=1$. Since $1-p^{k-1}$ is not an analytic function of $k$, hence we cannot take the derivative directly. But $\psi_{k}$ is an analytic function of $k$, so the derivative exists. This phenomenon in which the zero is forced by Euler factor is called trivial zero problem. Ferrero and Greenberg [4] considered the trivial zero problem for the first time in 1978 and found that the derivative has deep arithmetic meaning. The behavior of the derivative of some KubotaLeopoldt $p$-adic $L$-function with trivial zero has a deep relation with some arithmetic Iwasawa module (see [6]). The second such trivial zero phenomenon was found by Mazur et al. in [8], and then they conjectured that the derivative has a relation with $\tilde{\mathcal{L}}$ invariant. This conjecture was proved by Greenberg and Stevens in 1993 (see [7]). The function $\psi_{k}$ is very close to Coates-Wiles $k$ th derivative (see Section 7); actually, it only differs by the factor $\left(1-p^{k-1}\right)$, and was called Coates-Wiles homomorphism in de Shalit [3]. The question to find the derivative at $k=1$ of $\psi_{k}$ was proposed by Glenn Stevens in 1997. Simultaneously, we also tried to understand how the Bloch-Kato exponential map $\exp _{\mathbb{Q}_{p}(1)}$ can miss the Kummer class $\gamma_{p}$. Glenn Stevens predicted that the derivative of $\psi_{k}$ at 1 will give the Kummer class $\gamma_{p}$. We will prove this in this paper.

Let $\mathbb{C}_{p}$ denote the completion of $\overline{\mathbb{Q}}_{p}$. For a field $K \subset \mathbb{C}_{p}$, let $O_{K}$ denote the ring of integers. Choose Iwasawa's $\log : \mathbb{C}_{p}^{\times} \rightarrow \mathbb{C}_{p}$ such that $\log (p)=0$. In Section 2, we will review Fontaine's rings briefly and describe Bloch-Kato exponential map. In Section 3, we will define distributions and explain cohomology groups as Iwasawa module. In Section 4, we will introduce algebraic Fourier transformation and use Coleman power series to give some special distributions. In Section 5, we will review Perrin-Riou and Colmez theorems. In Section 6, we will show that Iwasawa's explicit reciprocity law 
is actually a special case of Perrin-Riou's theorem. In Section 7, we use the theory we developed so far to prove our theorem.

2. Fontaine's rings and Bloch-Kato exponential map. Let $\bar{O}=O_{\mathbb{C}_{p}} / p O_{\mathbb{C}_{p}}$. Let $\Re$ denote the projective limit of the diagram

$$
\bar{O} \leftarrow \bar{O} \leftarrow \bar{O} \leftarrow \cdots,
$$

where the transition maps are given by $x \rightarrow x^{p}$. The ring $\Re$ is a perfect ring with characteristic $p>0$ (see [5]). For $x \in \mathscr{R}, x=\left(x_{n}\right)_{n \in \mathbb{N}}$ satisfies $x_{n} \in \bar{O}$, and $x_{n+1}^{p}=x_{n}$. For each $n$, choose $\tilde{x}_{n} \in O_{\mathbb{C}_{p}}$ to be a representative of $x_{n}$. Then one can show that for each $m$, $\lim _{n \rightarrow \infty} \tilde{x}_{n+m}^{p^{n}}$ exists and the limit $x^{(m)}$ does not depend on the choices of the representatives. Hence, $x$ gives rise to a sequence $\left(x^{(m)}\right)_{m \in \mathbb{N}}$ in $O_{\mathbb{C}_{p}}$ such that $\left(x^{(m+1)}\right)^{p}=x^{(m)}$. On the other hand, if we have a sequence $\left(x^{(m)}\right)_{m \in \mathbb{N}}$ in $O_{\mathbb{C}_{p}}$ such that $\left(x^{(m+1)}\right)^{p}=x^{(m)}$, then $\left(\bar{x}^{(m)}\right)_{m \in \mathbb{N}}$ is an element in $\mathscr{R}$. Hence, $\mathscr{R}$ is in one-to-one correspondence with the set

$$
\left\{\left(x^{(m)}\right)_{m \in \mathbb{N}} \mid \forall m \in \mathbb{N}, x^{(m)} \in O_{\mathbb{C}_{p}},\left(x^{(m+1)}\right)^{p}=x^{(m)}\right\} .
$$

Define a function $v_{\mathscr{R}}: \Re \rightarrow \mathbb{Q} \cup\{\infty\}$ by

$$
v_{\mathscr{R}}\left(\left(x^{(m)}\right)_{m \in \mathbb{N}}\right):=v\left(x^{(0)}\right),
$$

where $v$ is the valuation of $\mathbb{C}_{p}$ such that $v(p)=1$. The ring $\mathscr{R}$ is complete with respect to $v_{\Re}$.

Let $W(\Re)$ denote the Witt vector ring of $\Re$. Recall that the underlying set of $W(\Re)$ is the set $\mathscr{R}^{\mathbb{N}}=\left\{\left(x_{0}, x_{1}, \ldots\right) \mid x_{i} \in \mathscr{R}\right\}$. The ring structure is given in terms of Witt polynomials (see [10]). Since $\bar{O}$ is an $\overline{\mathbb{F}}_{p}$-algebra, $W(\mathscr{R})$ is a $W\left(\overline{\mathbb{F}}_{p}\right)$-algebra. For $x \in \mathscr{R}$, let

$$
[x]:=(x, 0,0, \ldots) \in W(\mathscr{R})
$$

denote the Teichmüller representative of $x$. For $\left(x_{0}, x_{1}, \ldots, x_{n}, \ldots\right) \in W(\mathscr{R})$, we have the identity

$$
\left(x_{0}, x_{1}, \ldots, x_{n}, \ldots\right)=\left[x_{0}\right]+p\left[x_{1}\right]^{p^{-1}}+\cdots+p^{n}\left[x_{n}\right]^{p^{-n}}+\cdots,
$$

where for $x \in \mathscr{R},[x]^{p^{-1}}$ is the unique element $w$ of $W(\mathscr{R})$ such that $w^{p}=[x]$.

Let

$$
\theta: W(\Re) \rightarrow O_{\mathbb{C}_{p}}
$$

be defined by

$$
\theta\left(x_{0}, x_{1}, \ldots\right)=\sum_{n=0}^{\infty} p^{n} x_{n}^{(n)}
$$


Then it is easy to see that $\theta$ is a $\mathbb{Z}_{p}$-homomorphism and it is surjective. The Frobenius on $\mathscr{R}$ induces a continuous Frobenius map on $W(\mathscr{R})$ with respect to the product topology, we denote it by $\varphi$, which sends $\left(x_{0}, x_{1}, \ldots, x_{n}, \ldots\right)$ to $\left(x_{0}^{p}, x_{1}^{p}, \ldots, x_{n}^{p}, \ldots\right)$. The map $\varphi$ is an isomorphism, semilinear over $W\left(\overline{\mathbb{F}}_{p}\right)$. The ring $W(\mathscr{R})$ can also be endowed with $p$ adic topology and $I$-adic topology. Let $\varepsilon=\left(1, \zeta_{p}, \zeta_{p^{2}}, \ldots\right) \in \mathscr{R}$. The element $[\varepsilon] \in W(\mathscr{R})$ has the property $\theta([\varepsilon])=1$. The element $\varphi^{-1}([\varepsilon])=\left[\left(\zeta_{p}, \zeta_{p^{2}}, \ldots\right), 0,0, \ldots\right]$. Let $u=$ $([\varepsilon]-1) /\left(\varphi^{-1}[\varepsilon]-1\right)$. The kernel of $\theta$ is a principal ideal of $W(\mathscr{R})$, which is generated by $u$ [5].

We will use $B_{\mathrm{dR}}^{+}, B_{\mathrm{dR}}, A_{\text {crys }}, B_{\text {crys }}^{+}, B_{\text {crys }}, A_{\text {max }}$, and $B_{\max }$ from Colmez [2].

LEMMA 2.1. The following sequences are exact:

$$
\begin{aligned}
& 0 \longrightarrow \mathbb{Q}_{p} \longrightarrow B_{\max }^{\varphi=1} \stackrel{\mathrm{Fil}^{<0}}{\longrightarrow} B_{\mathrm{dR}} / B_{\mathrm{dR}}^{+} \longrightarrow 0 \\
& 0 \longrightarrow \mathbb{Q}_{p} \longrightarrow \mathrm{Fil}^{0} B_{\max } \stackrel{\varphi-1}{\longrightarrow} B_{\max } \longrightarrow 0
\end{aligned}
$$

where $\varphi$ is the Frobenius of $B_{\mathrm{dR}}$ which is induced by the one from $\mathscr{R}$.

Proof. See Colmez [2, Appendix A].

For a continuous $G_{\mathbb{Q}_{p}}$-representation $V$, finite-dimensional $\mathbb{Q}_{p}$-vector space, define $D_{\text {crys }}(V):=\left(B_{\text {crys }} \otimes V\right)^{G_{\mathbb{Q} p}}, D_{\mathrm{dR}}(V):=\left(B_{\mathrm{dR}} \otimes V\right)^{G_{\mathbb{Q} p}}$. Then $D_{\text {crys }}(V)$ is a finite-dimensional $\mathbb{Q}_{p}$-vector space, with a Frobenius action (acts on $V$ trivially) [5]. The operator $D_{\mathrm{dR}}$ has a filtration given by $\mathrm{Fil}^{i}\left(D_{\mathrm{dR}}(V)\right)=\left(B_{\mathrm{dR}}^{i} \otimes V\right)^{G_{\mathbb{Q} p}}$. The dimensions have the following relation:

$$
\operatorname{dim}_{\mathbb{Q}_{p}}\left(D_{\text {crys }}(V)\right) \leq \operatorname{dim}_{\mathbb{Q}_{p}}\left(D_{\mathrm{dR}}(V)\right) \leq \operatorname{dim}_{\mathbb{Q}_{p}}(V) .
$$

If $\operatorname{dim}_{\mathbb{Q}_{p}}\left(D_{\mathrm{dR}}(V)\right)=\operatorname{dim}_{\mathbb{Q}_{p}}(V)$, then $V$ is called a de Rham representation. If $\operatorname{dim}_{\mathbb{Q}_{p}}\left(D_{\text {crys }}(V)\right)=\operatorname{dim}_{\mathbb{Q}_{p}}(V)$, then $V$ is called a crystalline representation. Note that a crystalline representation must be a de Rham representation. In the following, all representations are assumed to be de Rham representations. Similarly, we can also define $D_{\max }(V):=\left(B_{\max } \otimes V\right)^{G_{\mathbb{Q}_{p}}}$; Colmez proved that this is the same as $D_{\text {crys }}(V)$. For a crystalline representation $V$, let $D(V)=D_{\text {crys }}(V)$.

For a de Rham representation $V$, taking tensor product with the exact sequence (2.8), we have the following exact sequence:

$$
0 \longrightarrow V \longrightarrow B_{\max }^{\varphi=1} \otimes V \longrightarrow B_{\mathrm{dR}} / B_{\mathrm{dR}}^{+} \otimes V \longrightarrow 0 ;
$$

taking the Galois cohomology, we have a map

$$
\left(B_{\mathrm{dR}} / B_{\mathrm{dR}}^{+} \otimes V\right)^{G_{\mathbb{Q} p}} \longrightarrow H^{1}\left(\mathbb{Q}_{p}, V\right)
$$

Then the Bloch-Kato exponential map

$$
\exp _{V}:\left(B_{\mathrm{dR}} \otimes V\right)^{G_{\mathbb{Q} p}} \longrightarrow H^{1}\left(\mathbb{Q}_{p}, V\right)
$$

is defined as the composition

$$
\left(B_{\mathrm{dR}} \otimes V\right)^{G_{\mathbb{Q} p}} \longrightarrow\left(B_{\mathrm{dR}} / B_{\mathrm{dR}}^{+} \otimes V\right)^{G_{\mathbb{Q} p}} \longrightarrow H^{1}\left(\mathbb{Q}_{p}, V\right) .
$$


The kernel of this map is $\operatorname{Fil}^{0} D_{\mathrm{dR}}(V)+D_{\text {crys }}(V)^{\varphi=1}$, and the image is $H_{e}\left(\mathbb{Q}_{p}, V\right):=$ $\operatorname{ker}\left\{H^{1}\left(\mathbb{Q}_{p}, V\right) \rightarrow H^{1}\left(\mathbb{Q}_{p}, B_{\text {crys }}^{\varphi=1} \otimes V\right)\right\}$.

For a Galois representation $V$, let $V(k)$ denote the $k$ th cyclotomic twist of $V$. That is, let $\chi$ denote the cyclotomic character, $\zeta_{p^{n}}^{\sigma}=\zeta_{p^{n}}^{\chi(\sigma)}$ for all $n \geq 1, V(k):=V\left(\chi^{k}\right)$.

Consider the example $V=\mathbb{Q}_{p}(1)=\mathbb{Q}_{p} \cdot e$; in this case, $D_{\mathrm{dR}}(V)=\mathbb{Q}_{p} \cdot(e / t)$ is a one-dimensional vector space, where $t=\log ([\varepsilon])$. The isomorphism $H^{1}\left(\mathbb{Q}_{p}, \mathbb{Q}_{p}(1)\right) \cong$ $\widehat{\mathbb{Q}_{p}^{x}} \otimes \mathbb{Q}_{p}$ is given by the Kummer map. To be more precise, it is generated by $\gamma_{1+p}, \gamma_{p}$, where, for $\alpha \in \mathbb{Q}_{p}^{\times}$,

$$
\gamma_{\alpha}: \tau \longrightarrow \log _{\varepsilon}\left(\ldots, \frac{\tau\left(\alpha^{1 / p^{n}}\right)}{\alpha^{1 / p^{n}}}, \ldots\right) \otimes e
$$

is the Kummer class. Hence, we have $H^{1}\left(\mathbb{Q}_{p}, \mathbb{Q}_{p}(1)\right) \cong \mathbb{Q}_{p}^{2}$, then the exponential map for $\mathbb{Q}_{p}$ is

$$
\exp _{\mathbb{Q}_{p}(1)}: D_{\mathrm{dR}}\left(\mathbb{Q}_{p}(1)\right) \longrightarrow H^{1}\left(\mathbb{Q}_{p}, \mathbb{Q}_{p}(1)\right) .
$$

LEMMA 2.2. It follows that

$$
\exp _{\mathbb{Q}_{p}(1)}\left(\frac{e}{t}\right)=\frac{\gamma_{1+p}}{\log (1+p)}
$$

Proof. In the exact sequence

$$
0 \longrightarrow \mathbb{Q}_{p} \longrightarrow B_{\max }^{\varphi=1} \longrightarrow B_{\mathrm{dR}} / B_{\mathrm{dR}}^{+} \longrightarrow 0
$$

$\log [\widetilde{1+p}] / t \cdot \log (1+p)$ maps to $1 / t$, so $(\log [\widetilde{1+p}] / t \cdot \log (1+p)) \otimes e$ maps to $(1 / t) \otimes e \in$ $D_{\mathrm{dR}}\left(\mathbb{Q}_{p}(1)\right)$, hence the class $\exp _{\mathbb{Q}_{p}(1)}(e / t)$ is represented by

$$
\begin{aligned}
& \tau \longrightarrow(\tau-1) \cdot\left(\frac{\log [\widetilde{1+p}]}{t \cdot \log (1+p)} \otimes e\right) \\
& =\frac{1}{t \log (1+p)}\left(\log \left(\left[\left(\ldots, \tau\left((1+p)^{1 / p^{n}}\right), \ldots\right), \ldots\right]\right)\right. \\
& \left.-\log \left(\left[\left(\ldots,(1+p)^{1 / p^{n}}, \ldots\right), \ldots\right]\right)\right) \otimes e \\
& =\frac{1}{t \log (1+p)}\left(\log \left(\left[\left(\ldots, \frac{\tau\left((1+p)^{1 / p^{n}}\right)}{(1+p)^{1 / p^{n}}}, \ldots\right), \ldots\right]\right)\right) \\
& =\frac{1}{t \log (1+p)}\left(\log \left[\varepsilon^{\gamma_{1+p}(\tau)}\right]\right) \\
& =\frac{\gamma_{1+p}(\tau)}{\log (1+p)} \text {. }
\end{aligned}
$$

For $k>1$, it is easy to see that $\operatorname{dim}_{\mathbb{Q}_{p}} D_{\mathrm{dR}}\left(\mathbb{Q}_{p}(k)\right)=\operatorname{dim}_{\mathbb{Q}_{p}} H^{1}\left(\mathbb{Q}_{p}, \mathbb{Q}_{p}(k)\right)=1$ and $\exp _{\mathbb{Q}_{p}(k)}$ is an isomorphism. In some sense, $\gamma_{p}$ and $\gamma_{1+p}$ should have the same positions in $H^{1}\left(\mathbb{Q}_{p}, \mathbb{Q}_{p}(1)\right)$. Note that for $k=1$, the left-hand side has dimension 1 and the righthand side has dimension 2 , so the image is a one-dimensional vector space, and $\gamma_{p}$ is not in the image. In this paper, we will show that the "derivative of Bloch-Kato map" is essentially $\gamma_{p}$. To be a little bit more precise, we need the following definitions. 
Let $\mathscr{X}=\operatorname{Hom}_{\text {cont }}\left(\mathbb{Z}_{p}^{\times}, \mathbb{C}_{p}^{\times}\right)$which is identical to $B\left(\mu_{p-1}, 1\right)$ and there is an obvious inclusion $\mathbb{Z} \subset \mathscr{X}$.

DEFINITION 2.3. Given $\mathscr{S} \subset \mathscr{X}$, a rigid analytic subspace over $\mathbb{Q}_{p}$, an analytic family of Galois representations over $\mathscr{S}$ is a pair $(V, \rho)$, where $(1) V$ is a de Rham representation of $G_{\mathbb{Q}_{p}},(2) \rho: \mathscr{Y} \times G_{\mathbb{Q}_{p}} \rightarrow \mathrm{Gl}_{\mathbb{Q}_{p}}(V)$ is continuous in $\sigma$ and is analytic in $k$.

DEFINITION 2.4. Let $(V, \rho)$ over $\mathscr{S}$ be a family of Galois representations of $G_{\mathbb{Q}_{p}}$ and let $V_{k}$ denote the Galois representation of $G_{\mathbb{Q}_{p}}$ such that the underlying space is $V$ and the action is given by

$$
\sigma \circ v=\rho_{k}(\sigma)(\sigma(v))
$$

A family of classes $\xi_{k} \in H^{1}\left(\mathbb{Q}_{p}, V_{k}\right)$ is said to be an analytic family if there is a cocycle representation $\sigma \rightarrow \xi_{k}(\sigma)$ such that for all $\sigma \in G_{\mathbb{Q}_{p}}, \xi_{k}(\sigma)$ is an analytic function of $k$.

Now, we can go back to answer the question on $\gamma_{p}$. In Section 7 , we will show that $\psi_{k}=\left(\left(1-p^{1-k}\right) /\left(1-p^{-k}\right)\right)(k-1) ! \exp _{V_{k}}\left(1_{k}\right)$ is an analytic family of cohomology classes in $H^{1}\left(\mathbb{Q}_{p}, \mathbb{Q}_{p}(k)\right)$ and $\left.(d / d k)\left(\psi_{k}\right)\right|_{k=1}=-\left(1-p^{-1}\right)^{-1} \gamma_{p}$. In other words, $\gamma_{p}$ appears in the first coefficient of the "Taylor expansion" of Bloch-Kato exponential map.

3. Distributions and Iwasawa module. Let $I \subset \mathbb{Z}$ be a subset and let $L P^{I}=\left\{x^{k}\right.$. $\left.1_{a+p^{n} \mathbb{Z}_{p}} \mid k \in I, a \in \mathbb{Q}_{p}\right\}$. An algebraic $I$-distribution with values in $M$ is a finitely additive function $\mu: L P^{I} \rightarrow M$. Let $\mathscr{D}_{\text {alg }}^{I}\left(\mathbb{Q}_{p}, M\right)$ denote all the algebraic $I$-distributions with values in $M$. For $X \subset \mathbb{Q}_{p}$, a compact open subset, let $L P^{I}(X)=\left\{x^{k} \cdot 1_{\left(a+p^{n} \mathbb{Z}_{p} \cap \cap X\right.}\right\}$, then $\mathscr{D}_{\text {alg }}^{I}(X, M)$ is defined with respect to these test functions. Especially, we have $\mathscr{D}_{\text {alg }}^{I}\left(\mathbb{Z}_{p}^{\times}, M\right), \mathscr{D}_{\text {alg }}^{I}\left(\mathbb{Z}_{p}, M\right)$. Let $\mathscr{D}_{\text {alg }}^{+}\left(\mathbb{Q}_{p}, M\right)$ (resp., $\left.\mathscr{D}_{\text {alg }}^{-}\left(\mathbb{Q}_{p}, M\right)\right)$ denote the case $I=\mathbb{N}$ (resp., $I=-\mathbb{N}$ ). Note that when we say $\mathbb{N}$ we always mean $\mathbb{N}=\{0,1,2, \ldots\}$.

Let $L A=\left\{\right.$ locally analytic compactly supported functions in $\mathbb{Q}_{p}$ with values in $\left.\mathbb{Q}_{p}\right\}$. Let $L A^{\prime}=\left\{f: \mathbb{Q}_{p} \backslash\{0\} \rightarrow \mathbb{Q}_{p} \mid f\right.$ is locally analytic and compact supported such that thereexists $\left.N \in \mathbb{N}, x^{N} f \in L A\right\} . L A$ and $L A^{\prime}$ have Morita topology.

We let $A_{n}(X)$ denote the $\mathbb{Q}_{p}$-affinoid algebra of $B\left[X, p^{-n}\right]$. In particular, $A_{n}(X)$ is a Banach algebra under the Gauss norm. For a $p$-adic Banach space $A$, let $\mathscr{D}_{\text {cont }}\left(\mathbb{Q}_{p}, A\right):=$ $\{\mu: L A \rightarrow A \mid \mu$ is linear and continuous with respect to Morita topology . Note that $\mu$ is continuous if and only if it is continuous when restricted on each $\mathscr{A}_{n}(X), n \in \mathbb{Z}, X$ open.

DEFINITION 3.1. (a) Let $\mu \in \mathscr{D}_{\text {cont }}(Y, A)$. For each $n \in \mathbb{Z}$ and every compact open subset $X$ of $Y$, define $\|\mu\|_{\mathscr{A}_{n}(X)}$ to be the norm of the continuous linear function $\mu$ : $\mathscr{A}_{n}(X) \rightarrow A$ obtained by restricting $\mu$ to $\mathscr{A}_{n}(X)$.

(b) Similarly, if $\mu \in \mathscr{D}_{\text {alg }}^{I}(Y, A)$, then for each $n \in \mathbb{Z}$ and every compact open subset $X$ of $Y$, define $\|\mu\|_{L P_{n}^{I}(X)}$ to be the norm of the continuous linear function $\mu: L P^{I}(X) \cap$ $\mathscr{A}_{n}(X) \rightarrow A$ obtained by restricting $\mu$ to $L P^{I}(X) \cap \mathscr{A}_{n}(X)$. If $X \subset \mathbb{Q}_{p}$ is compact, then actually

$$
\|\mu\|_{\mathscr{A}_{n}(X)}=\sup _{a \in X, j \geq 0}\left\|\int_{a+p^{n} \mathbb{Z}_{p}}\left(\frac{x-a}{p^{n}}\right)^{j} \mu\right\| .
$$


Definition 3.2. For $r \in \overline{\mathbb{R}}^{+}, \mu \in \mathscr{D}_{\text {cont }}\left(\mathbb{Q}_{p}, A\right)$ is said to be tempered of order $r$ if for every compact open subset $X \subset \mathbb{Q}_{p}, p^{-[n r]}\|\mu\|_{\mathscr{A}_{n}(X)}$ is $r$-bounded. Let $\mathscr{D}_{r}\left(\mathbb{Q}_{p}, A\right) \subset$ $\mathscr{D}_{\text {cont }}\left(\mathbb{Q}_{p}, A\right)$ denote the set of distributions of order $r$. For $r_{1}<r_{2}$, $\mathscr{D}_{r_{1}}\left(\mathbb{Q}_{p}, A\right)$ $\subset \mathscr{D}_{r_{2}}\left(\mathbb{Q}_{p}, A\right)$. Let $\mathscr{D}_{\text {temp }}\left(\mathbb{Q}_{p}, A\right)=\cup_{r \geq 0} \mathscr{D}_{r}\left(\mathbb{Q}_{p}, A\right)$ denote all tempered distributions with values in $A$. From the above remark we see that $\mu$ is $r$-bounded if and only if

$$
p^{-[n r]} \sup _{a \in X, j \geq 0}\left\|\int_{a+p^{n} \mathbb{Z}_{p}}\left(\frac{x-a}{p^{n}}\right)^{j} \mu\right\|
$$

is $r$-bounded. A distribution with order $r$ is also called an $r$-admissible distribution.

LEMMA 3.3. For $\mu \in \mathscr{D}_{\text {cont }}\left(\mathbb{Q}_{p}, A\right), \mu$ has order $r$ if and only if for all $X$ compact open, all $x \in X, 0 \leq j \leq r$,

$$
p^{-[n r]} \sup _{a \in X, 0 \leq j \leq r}\left\|\int_{a+p^{n} \mathbb{Z}_{p}}\left(\frac{x-a}{p^{n}}\right)^{j} \mu\right\|
$$

is $r$-bounded.

Proof. Since $j>r$, then $p^{[n(j-r)]}\left\|\int_{a+p^{n} \mathbb{Z}_{p}}(x-a)^{j}\right\|$ tends to zero when $n \rightarrow \infty$.

If $V$ is a crystalline representation of $G_{\mathbb{Q}_{p}}$, we have a twist map

$$
\mathscr{D}_{\text {cont }}\left(\mathbb{Q}_{p}, D(V)\right) \stackrel{T w}{\longrightarrow} \mathscr{D}_{\text {cont }}\left(\mathbb{Q}_{p}, D(V(-1))\right)
$$

which sends $\mu$ to $(-t x) \mu$.

LEMMA 3.4. The kernel $\operatorname{ker}(T w)=\delta_{0} \otimes D(V), T w$ is surjective.

Proof. Obviously, we have $T w\left(\delta_{0} \otimes D(V)\right)=0$. If $\mu \in \operatorname{ker}(T w)$, then $\operatorname{supp}(\mu)=\{0\}$. Let $\mu_{1}=\mu-\left(\int \mu\right) \delta_{0}$, then $\int f(x) \mu_{1}=\int f(x) \mu-\left(\int \mu\right) \cdot f(0)=f(0) \cdot\left(\int \mu\right)-\left(\int \mu\right) \cdot f(0)=0$, hence $\mu=\left(\int \mu\right) \otimes \delta_{0}$.

For the surjectivity, given $v \in \mathscr{D}_{\text {cont }}\left(\mathbb{Q}_{p}, D(V(-1))\right)$, define $\omega \in \mathscr{D}_{\text {cont }}\left(\mathbb{Q}_{p}, D(V)\right)$ such that

$$
\int f \omega=\left(-t^{-1}\right) \int \frac{f-f(0) \cdot 1_{\mathbb{Z} p}}{x} v
$$

then $\int f(x)(-t x) \omega=\int f v$, hence $(-t x) \omega=v$.

For $\mu \in \mathscr{D}_{\text {alg }}^{I}\left(\mathbb{Q}_{p}, A\right)$, define an operator $\varphi_{\mathscr{D}}$ as

$$
\int_{\mathbb{Q}_{p}} f(x) \varphi_{\circledast} \mu:=\int_{\mathbb{Q}_{p}} f(p x) \mu
$$

If $A$ is a Dieudonne module, then $\varphi$ can act on it, hence both $\varphi$ and $\varphi_{\mathscr{D}}$ can act on $\mathscr{D}_{\text {alg }}^{I}\left(\mathbb{Q}_{p}, A\right)$. Then we define $\Phi=\varphi_{\mathscr{D}} \otimes \varphi$.

LEMMA 3.5. The twist map Tw induces a map

$$
\mathscr{D}_{\text {cont }}\left(\mathbb{Q}_{p}, D(V)\right)^{\Phi=1} \longrightarrow \mathscr{D}_{\text {cont }}\left(\mathbb{Q}_{p}, D(V(-1))\right)^{\Phi=1}
$$


with kernel $=\delta_{0} \otimes D(V)^{\varphi=1}$, image $=\left\{v \in \mathscr{D}_{\text {cont }}\left(\mathbb{Q}_{p}, D(V(-1))\right)^{\Phi=1} \mid \int_{\mathbb{Z}_{p}^{\times}} x^{-1} v=0\right\}$, and cokernel $=D(V(-1)) /(\varphi-p) D(V(-1)) \cong D(V) /(\varphi-1) D(V)$.

Proof. Assume that $\delta_{0} \otimes d$ is in the kernel, $\Phi\left(\delta_{0} \otimes d\right)=\delta_{0} \otimes d$. For all $f$, we have $\int f \Phi\left(\delta_{0} \otimes d\right)=\int f \delta_{0} \otimes d=f(0) \otimes d$, that is, $\varphi(f(0) \otimes d)=f(0) \otimes d$, hence $d \in D(V)^{\varphi=1}$.

Now, we calculate the image. If $v=T w(\mu)=(-t x) \mu$, then from the Colman-Colmez exact sequence [2], we have $\int_{\mathbb{Z}_{p}^{\times}} x^{-1} v=\int_{\mathbb{Z}_{p}^{\times}}(-t) \mu=0$. On the other hand, if $v$ satisfies $\int_{\mathbb{Z}_{p}^{\times}} x^{-1} v=0, \omega$ maps to $v$ from Lemma 3.4, we need to show that $\Phi(\omega)=\omega$. That is, for all $f, \int f \cdot \Phi(\omega)=\int f \omega$. The calculation shows that

$$
\begin{aligned}
\int f \Phi & (\omega)-\int f \omega \\
& =\varphi\left(\int f(p x) \omega\right)-\int f \omega \\
& =\varphi\left(\int \frac{f(p x)-f(0) \cdot 1_{\mathbb{Z}_{p}}}{(-t x)} v\right)-\int f \omega \\
& =(-t)^{-1} \int \frac{f(x)-f(0) \cdot 1_{\mathbb{Z}_{p}}(x / p)}{x} v-(-t)^{-1} \int \frac{f(x)-f(0) \cdot 1_{\mathbb{Z}_{p}}}{x} v \\
& =(-t)^{-1} \int \frac{1_{\mathbb{Z}_{p}^{x}} \cdot f(0)}{x} v=0 .
\end{aligned}
$$

The statement about cokernel follows immediately.

Define $\tilde{D}_{\text {temp }}\left(\mathbb{Q}_{p}, D(V)\right):=\varliminf_{\lim } \mathscr{D}_{\text {temp }}\left(\mathbb{Q}_{p}, D(V)\right)^{\Phi=1}$, where the transition maps are given by the above twist map.

LEMMA 3.6. For $\mu \in \mathscr{D}_{\text {cont }}\left(\mathbb{Z}_{p}^{\times}, A\right), \mu$ has order $r$ if and only if $x \mu$ has order $r$.

Proof. Assume that $\mu$ has order $r$ with $r \in \mathbb{R}$, then there is a constant $C>0$ such that for all $j \geq 0,\left\|\int_{a+p^{n} \mathbb{Z}_{p}}(x-a)^{j} \mu\right\| \leq C p^{[n(r-j)]}$, hence $\left\|\int_{a+p^{n} \mathbb{Z}_{p}} x(x-a)^{j} \mu\right\|=$ $\left\|\int_{a+p^{n} \mathbb{Z}_{p}}(x-a)^{j+1} \mu-p^{[n(r-j)]} \int_{a+p^{n} \mathbb{Z}_{p}} a(x-a)^{j} \mu\right\| \leq C p^{[n(r-j)]}$. If $r \notin \mathbb{R}$, then we take that $C=C_{n}$ tends to zero.

If $\mu$ has order $r$, by using the expansion $\int_{a+p^{n} \mathbb{Z}_{p}}(x-a)^{r}(\mu / x)=\int_{a+p^{n} \mathbb{Z}_{p}}(x-a)^{r}(1 /(a+$ $(x-a))) \mu=\int_{a+p^{n} \mathbb{Z}_{p}}(x-a)^{r} \cdot 1 / a \cdot \sum_{k \geq 0}((x-a) / a)^{k} \mu$, we see that $\left\|\int_{a+p^{n} \mathbb{Z}_{p}}(x-a)^{r}(\mu / x)\right\|$ $\leq C p^{[n(r-j)]}$, this proves the lemma.

For $\mu \in \mathscr{D}_{\text {cont }}\left(\mathbb{Z}_{p}, \mathbb{C}_{p}\right)$, define the Amice transformation

$$
\mathscr{A}_{\mu}(T)=\int_{\mathbb{Z} p}(1+T)^{x} \mu \in \mathbb{C}[[T]]
$$

DEFINITION 3.7. A formal power series $f(T)=\sum a_{n} T^{n} \in \mathbb{C}_{p}[[T]]$ is said to be of order $r$ if $p^{[n r]} a_{n}$ is $r$-bounded.

LEMmA 3.8. A distribution $\mu \in \mathscr{D}_{\text {cont }}\left(\mathbb{Z}_{p}, \mathbb{C}_{p}\right)$ has order $r$ if and only if $\mathscr{A}_{\mu}(T)$ has order $r$.

Proof. See [1]. 
4. Fourier transformation and Coleman power series. Recall that we fixed $\zeta_{p^{n}}$ which is a $p^{n}$ th root of unity. Let $\varepsilon_{n}:=\left(\zeta_{p^{n}}, \zeta_{p^{n+1}}, \ldots\right) \in \mathscr{R}$, note that $\varepsilon_{n}^{p^{n}}=\varepsilon$, [ $\left.\varepsilon_{n}\right] \in$ $W(\mathscr{R})$. For $x \in \mathbb{Q}_{p}, x=p^{-n} \cdot y$ with some $n \in \mathbb{N}$ and $y \in \mathbb{Z}_{p}$, define $\varepsilon^{x}:=\varepsilon_{n}^{y} \in \mathscr{R}$. Obviously, this is well defined, and we get an element $\left[\varepsilon^{x}\right] \in W(\mathscr{R})$. For $x \in \mathbb{Q}_{p}$, $\exp (t x)=\sum_{k=0}^{+\infty}\left((t x)^{k} / k !\right)$ converges. Define $\varepsilon(x):=\left[\varepsilon^{x}\right] / \exp (t x)$ for $x \in \mathbb{Q}_{p}$. Then $\varepsilon(x)$ has the following properties:

(i) if $x \in p^{-n} \mathbb{Z}_{p}^{\times}$, with $n \geq 0$, then $\varepsilon(x)$ is a $p^{n}$ th root of unity, $\varepsilon(x)=1$ if and only if $x \in \mathbb{Z}_{p}$. Moreover, $\varepsilon\left(1 / p^{n}\right)=\zeta_{p^{n}}$;

(ii) it follows that

$$
\sum_{x=0}^{p^{n}-1} \varepsilon\left(\frac{a x}{p^{n}}\right)= \begin{cases}p^{n}, & \text { if } a \equiv 0\left(\bmod p^{n}\right), \\ 0, & \text { otherwise for } a \in \mathbb{Z}_{p} \backslash p^{n} \mathbb{Z}_{p}\end{cases}
$$

(iii) for $x, y \in \mathbb{Q}, \varepsilon(x+y)=\varepsilon(x) \varepsilon(y)$;

(iv) for a cyclotomic character $\chi, \sigma(\varepsilon(x))=\varepsilon(\chi(\sigma) x)$.

If $f$ is a locally constant function with compact support in $\mathbb{Q}_{p}$, define

$$
\mathscr{F}_{\mathrm{F}} \mathrm{alg}(f)(y):=\int_{\mathbb{Q}_{p}} f(x) \varepsilon(x y) \mu_{\text {Haar }}(x),
$$

where $\mu_{\text {Haar }} \in \mathscr{D}_{\text {naive }}\left(\mathbb{Q}_{p}, \mathbb{Q}_{p}\right)$ such that $\mu_{\text {Haar }}\left(a+p^{n} \mathbb{Z}_{p}\right)=1 / p^{n}$. Since $f$ is locally constant, this means that we can find an $m$ such that on $a+p^{m} \mathbb{Z}_{p}, f$ is constant, hence the integral equals

$$
\sum_{a} \int_{a+p^{m} \mathbb{Z}_{p}} f(x) \varepsilon(x y) \mu_{\text {Haar }}(x)=\frac{1}{p^{m}} \sum_{a \bmod p^{m}} f(a) \sum_{x \in a+p^{m} \mathbb{Z}_{p}} \varepsilon(x y) .
$$

From property (ii) of $\varepsilon(x)$, if $y$ is outside of $p^{-m} \mathbb{Z}_{p}$, then this sum is zero, hence $\mathscr{F}_{\text {alg }}(f)$ is well defined and compactly supported. On the other hand, since $f$ is compactly supported, we can assume that $f$ is supported on $p^{-m} \mathbb{Z}_{p}$ for some $m$. Since $\varepsilon\left(p^{-m} y\right)$ is locally constant, this implies that $\mathscr{F}_{\text {alg }}(f)$ is locally constant. Extend the above definition to test function $\left\{x^{k} \cdot 1_{a+p^{n} \mathbb{Z}_{p}}, k \geq 0\right\}$, define

$$
\mathscr{F}_{\mathrm{alg}}\left(f^{\prime}\right)(y):=(-t y) \mathscr{F}_{\mathrm{alg}}(f)(y) .
$$

Proposition 4.1. The Fourier transformation $\mathscr{F}_{\text {alg }}$ enjoys the following properties:

(i) $\mathscr{F}_{\mathrm{alg}}(f(x+a))(y)=\varepsilon(-a y) \mathscr{F}_{\mathrm{alg}}(f)(y)$, for $a \in \mathbb{Q}_{p}$,

(ii) $\mathscr{F}_{\text {alg }}(\varepsilon(a x) f(x))(y)=\mathscr{F}_{\text {alg }}(f)(y+a)$,

(iii) $\mathscr{F}_{\mathrm{F}} \mathrm{alg}(f(c x))(y)=|c|^{-1} \mathscr{F}_{\mathrm{F}} \mathrm{alg}(f)\left(c^{-1} y\right)$,

(iv) $\mathscr{F}_{\text {alg }}\left(x^{k} \cdot 1_{a+p^{n} \mathbb{Z}_{p}}\right)(y)=p^{-n} \cdot\left(k ! /(-t y)^{k}\right) \varepsilon(a y) 1_{p^{-n} \mathbb{Z}_{p}}(y)$ if $k \geq 0, n \in \mathbb{Z}$, $a \in \mathbb{Q}_{p}$,

(v) $\mathscr{F}_{\text {alg }} \circ \mathscr{F}_{\text {alg }}(f)(y)=f(-y)$.

Proof. The properties follow easily from the definitions.

For $h \in \mathbb{Z}$, define the twist for $\mathscr{F}_{\text {alg }}$ as

$$
\mathscr{F}_{\mathrm{alg}}^{(h)}(f):=(-t y)^{h-1} \mathscr{F}_{\mathrm{alg}}\left(x^{h-1} f(x)\right)(y),
$$


where $f \in L P^{[1-h,+\infty)}$, then we have

$$
\mathscr{F}_{\text {alg }}^{(h)}\left(x^{k} \cdot 1_{a+p^{n} \mathbb{Z}_{p}}\right)(y)=p^{-n} \frac{(k+h-1) !}{(-t y)^{k}} \varepsilon(a y) 1_{p^{-n} \mathbb{Z}_{p}}(y)
$$

for all $k \geq 1-h, n \in \mathbb{Z}, a \in \mathbb{Q}_{p}$.

Now, we define the algebraic Fourier transformation on distributions as follows. For $\mu \in \mathscr{D}_{\text {alg }}^{(-\infty, h-1]}\left(\mathbb{Q}_{p}, D(V)\right)$, define $\mathscr{F}_{\text {alg }}^{(h)}(\mu)$ such that

$$
\int_{\mathbb{Q}_{p}} f(x) \mathscr{F}_{\mathrm{alg}}^{(h)}(\mu):=\int_{\mathbb{Q}_{p}} \mathscr{F}_{\mathrm{alg}}^{(h)}(f) \mu .
$$

For $\alpha \in \mathbb{Z}_{p}^{\times}$, let $\pi=p \alpha$. Let $f_{\pi}(x) \in \mathbb{Z}_{p}[[x]]$ be a Frobenius corresponding to $\pi$, so $f_{\pi}(x) \equiv \pi x(\bmod \operatorname{deg} 2)$ and $f_{\pi}(x) \equiv x^{p}(\bmod p)$. Let $\mathcal{f}$ be the one-dimensional LubinTate formal group over $\mathbb{Z}_{p}$ corresponding to $f_{\pi}$ and let $[+]$ denote the formal addition. Let $W_{\pi}^{n}:=\left\{x \in \mathbb{C}_{p} \mid f_{\pi}^{(n)}(x)=0\right\}, K_{n}=\mathbb{Q}_{p}\left(W_{\pi}^{n}\right)$, and $K_{\infty}=\cup_{n \geq 1} K_{n}$. Hence, $K_{\infty} / \mathbb{Q}_{p}$ is a totally ramified extension with Galois group $\mathbb{Z}_{p}^{\times}$. We call this tower the Lubin-Tate tower corresponding to the formal group $\mathfrak{f}$. Let $R=\mathbb{Z}_{p}[[T]]$ and $U=\underline{\lim } O_{K_{n}}^{\times}$, where the map is with respect to the norm map. Assume that $\beta \in \mathcal{U}$, then Coleman's theorem tells us that there is a unique (Coleman) power series $\mathfrak{g}_{\beta} \in \mathbb{Z}_{p}[[T]]$ such that

(i) $g_{\beta}\left(\omega_{i}\right)=\beta_{i}$ for all $i \geq 1$,

(ii) $g_{\beta}^{\varphi} \circ f_{\pi}(x)=\prod_{w \in W_{\pi}^{1}} g_{\beta}(x[+] w)$.

Assume that $\beta \in \mathcal{U} \operatorname{such}$ that $\beta_{n} \equiv 1\left(\bmod \omega_{n}\right)$. Then $g_{\beta}(T) \equiv 1 \bmod (\mathfrak{p}, T)$, hence we can define

$$
\widetilde{\log g_{\beta}}(T):=\log g_{\beta}(T)-\frac{1}{p} \sum_{w \in W_{\pi}^{1}} \log g_{\beta}(T[+] w)
$$

The property (ii) of the Coleman power series implies that $\widetilde{\log } g_{\beta}(T)$ has integral coefficients. Define an algebraic distribution $\mu_{\beta} \in \mathscr{D}_{\text {alg }}^{+}\left(\mathbb{Z}_{p}, \widehat{\mathbb{Q}_{p}^{u r}}\right)$ such that

$$
\int_{\mathbb{Z}_{p}}(1+T)^{x} \mu_{\beta}(x)=\log g_{\beta} \circ \eta(T) .
$$

Proposition 4.2. (i) The restriction of $\mu_{\beta}$ to $\left.\mathbb{Z}_{p}^{\times} \mu_{\beta}\right|_{\mathbb{Z}_{p}^{\times}}$is a measure and its Amice transformation is $\widetilde{\log } g_{\beta} \circ \eta(T)$.

(ii) The distribution $\left.\mu_{\beta}\right|_{\mathbb{Z}_{p}^{\times}}$is a measure in $\mathscr{D}_{1}\left(\mathbb{Q}_{p}, \widehat{\mathbb{Q}_{p}^{u r}}\right)^{\Phi=1}$ and has the following Galois property:

$$
\sigma\left(\int_{\mathbb{Q}_{p}} f(x) \mu_{\beta}\right)=\int_{\mathbb{Q}_{p}} f(\psi(\sigma) x) \mu_{\beta} \quad \forall \sigma
$$

for all $f(x): \mathbb{Q}_{p} \rightarrow \mathbb{Q}_{p}$.

Proof. It is easy to see that

$$
\int_{\mathbb{Z}_{p}^{\times}}(1+T)^{x} \mu_{\beta}=\int_{\mathbb{Z}_{p}}(1+T)^{x} \mu_{\beta}-\int_{p \mathbb{Z} p}(1+T)^{x} \mu_{\beta} .
$$


By property (ii),

$$
g_{\beta} \circ f_{\pi}(X)=\prod_{w \in W_{\pi}^{1}} g_{\beta}(X[+] w)
$$

let $X=\eta(T)$, then

$$
\begin{aligned}
g_{\beta} \circ f_{\pi}(\eta(T)) & =\prod_{\zeta \in \mu_{p}} g_{\beta}(\eta(T)[+] \eta(\zeta-1)) \\
& =\prod_{\zeta \in \mu_{p}} g_{\beta} \eta(\zeta(1+T)-1) .
\end{aligned}
$$

By using $f_{\pi} \circ \eta=\eta^{\varphi} \circ[p]$, we see that

$$
\left(g_{\beta} \circ \eta\right)^{\varphi} \circ[p]=\prod_{\zeta}\left(g_{\beta} \eta\right)(\zeta(1+T)-1) ;
$$

taking logarithm, and using the definition for $\mu_{\beta}$, we have

$$
\varphi\left(\int_{\mathbb{Z}_{p}}(1+[p] T)^{x} \mu_{\beta}\right)=\sum_{\zeta} \int_{\mathbb{Z}_{p}} \zeta^{x}(1+T)^{x} \mu_{\beta}=p \int_{p \mathbb{Z}_{p}}(1+T)^{x} \mu_{\beta} .
$$

Hence,

$$
\begin{aligned}
\int_{\mathbb{Z}_{p}^{\times}}(1+T)^{x} \mu_{\beta} & =\log g_{\beta} \eta(T)-\frac{1}{p} \varphi\left(\log g_{\beta} \circ \eta^{\varphi} \circ([p] T)\right) \\
& =\log g_{\beta} \circ \eta-\frac{1}{p} \log g_{\beta} \circ f_{\pi} \circ \eta(T) \\
& =\widetilde{\log } g_{\beta} \circ \eta(T)
\end{aligned}
$$

has integral coefficients, hence $\left.\mu_{\beta}\right|_{\mathbb{Z}_{p}^{\times}}$is a measure.

To prove the second property, since

$$
\eta(T): \mathbb{G}_{m} \longrightarrow \mathscr{F}_{\pi},
$$

by comparing the values at $T_{n}=\zeta_{p^{n}}-1$, we can show that

$$
\sigma(\eta(T))=\eta\left((1+T)^{\psi(\sigma)}-1\right) \quad \forall \sigma \in G_{\mathbb{Q}_{p}}
$$

From this property, we see that

$$
\begin{aligned}
\sigma\left(\int_{\mathbb{Z}_{p}}(1+T)^{x} \mu_{\beta}\right) & =\sigma\left(\log g_{\beta} \circ \eta(T)\right) \\
& =\log g_{\beta} \circ \sigma(\eta(T)) \\
& =\log g_{\beta} \circ \eta\left((1+T)^{\psi(\sigma)}-1\right) \\
& =\int_{\mathbb{Z}_{p}}(1+T)^{\psi(\sigma) x} \mu_{\beta},
\end{aligned}
$$


so for general $f$, we have

$$
\sigma\left(\int_{\mathbb{Z}_{p}} f(x) \mu_{\beta}\right)=\int_{\mathbb{Z}_{p}} f(\psi(\sigma) x) \mu_{\beta}
$$

by extending $\mu_{\beta}$ to $\mathbb{Q}_{p}$, we have for all $f$,

$$
\sigma\left(\int_{\mathbb{Q} p} f(x) \mu_{\beta}\right)=\int_{\mathbb{Q} p} f(\psi(\sigma) x) \mu_{\beta}
$$

To show that $\mu_{\beta}$ is 1 -admissible, by definition and Lemma 3.3, we only need to show that $p^{n(1-j)} \int_{a+p^{n} \mathbb{Z}_{p}}(x-a)^{j} \mu_{\beta}$ is $r$-bounded for $j=0,1$. For $j=0$, if $a \neq 0$, then since $\left.\mu_{\beta}\right|_{\mathbb{Z}_{p}^{\times}}$ is a measure, the integral $p^{n} \int_{a+p^{n} \mathbb{Z}_{p}} \mu_{\beta}$ is always bounded. If $a=0$, then $p^{n} \int_{p^{n} \mathbb{Z}_{p}} \mu_{\beta}=$ $\varphi^{n}\left(\int_{\mathbb{Z}_{p}} \mu_{\beta}\right)=\varphi^{n} \log \mathcal{g}_{\beta}(0)=\log \mathcal{g}_{\beta}(0)$, hence, bounded.

For $j=1$, if $a \neq 0$, then $\int_{a+p^{n} \mathbb{Z}_{p}} x \mu_{\beta}$ is bounded. If $a=0$, then $\int_{p^{n} \mathbb{Z}_{p}} x \mu_{\beta}=\varphi^{n}\left(\int_{\mathbb{Z}_{p}} x \mu_{\beta}\right)=$ $\left.\varphi^{n}\left(\Omega \cdot g_{\beta}^{\prime}(0) / g_{\beta}(0)\right)=\alpha^{n} \Omega\left(g_{\beta}^{\prime}(0)\right) / g_{\beta}(0)\right)$, hence, bounded.

5. Perrin-Riou and Colmez theorems. Let $K_{n}=\mathbb{Q}_{p}\left(\zeta_{p^{n}}\right)$ and $K_{\infty}=\cup_{n \geq 1} K_{n}$. Let $\Gamma=$ $\operatorname{Gal}\left(K_{\infty} / \mathbb{Q}_{p}\right), \chi: \Gamma \cong \mathbb{Z}_{p}^{\times}$be the cyclotomic character. For $x \in K_{\infty}$ and $n \in \mathbb{N}$, define $T_{n}(x)=\left(1 / p^{m}\right) \operatorname{Tr}_{K_{m} / K_{n}}(x)$ for $m \gg 1$. For a crystalline representation $V$, that is, a finite-dimensional $\mathbb{Q}_{p}$-vector space such that $G_{\mathbb{Q}_{p}}$ has a continuous action on it and $V$ is crystalline, let $D(V):=D_{\text {crys }}(V)$ denote the Dieudonne module of $V$. Then from Colmez [2], $T_{n}$ can be extended to $B_{\mathrm{dR}}^{G_{K_{\infty}}} \otimes D(V)$. Then it is known that $D(V)$ has a Frobenius endomorphism and a filtration which we denote by $\mathrm{Fil}^{i} D(V)$. This filtration is decreasing, separated, and exhausted. That is,

$$
\operatorname{Fil}^{i} D(V) \supseteq \mathrm{Fil}^{i+1} D(V), \quad \cap_{i} \operatorname{Fil}^{i} D(V)=\{0\}, \quad \cup_{i} \mathrm{Fil}^{i} D(V)=D(V) .
$$

If $F \in K_{\infty}((t)) \otimes D(V), F=\sum_{k \gg-\infty} t^{k} d_{k}$ with $d_{k} \in K_{\infty} \otimes D(V)$, define $\delta_{V(-k)}(F)$ to be $t^{k} d_{k}$. For $I \subset \mathbb{Z}$, we have the algebraic distribution $\mathscr{D}_{\text {alg }}^{I}\left(\mathbb{Q}_{p}, D(V)\right)$ from Section 3. For $h \in \mathbb{Z}$, we defined the algebraic Fourier transformation $\mathscr{F}_{\text {alg }}^{(h)}: \mathscr{D}_{\text {alg }}^{(-\infty, h-1]}\left(\mathbb{Q}_{p}, D(V)\right) \rightarrow$ $\mathscr{D}_{\mathrm{alg}}^{[1-h,+\infty)}\left(\mathbb{Q}_{p}, B_{\mathrm{dR}} \otimes V\right)$ as

$$
\int_{\mathbb{Q}_{p}} f(x) \mathscr{F}_{\text {alg }}^{(h)}(\mu):=\int_{\mathbb{Q}_{p}} \mathscr{F}_{\text {alg }}^{(h)}(f) \mu,
$$

then Perrin-Riou and Colmez proved that the image is fixed by $G_{\mathbb{Q}_{p}}$, and the Perrin-Riou exponential map $\operatorname{Exp}_{h, V}$ is defined as the composition of the following maps:

$$
\begin{aligned}
\mathscr{D}_{\mathrm{alg}}^{(-\infty, h-1]}\left(\mathbb{Q}_{p}, D(V)\right) & \longrightarrow \mathscr{D}_{\mathrm{alg}}^{[1-h,+\infty)}\left(\mathbb{Q}_{p}, B_{\mathrm{dR}} \otimes V\right)^{G_{\mathbb{Q} p}} \\
& \longrightarrow \mathscr{D}_{\mathrm{alg}}^{[1-h,+\infty)}\left(\mathbb{Z}_{p}^{\times}, B_{\mathrm{dR}} / B_{\mathrm{dR}}^{+} \otimes V\right)^{G_{\mathbb{Q} p}} \\
& \longrightarrow H^{1}\left(\mathbb{Q}_{p}, \mathscr{D}_{\mathrm{alg}}^{[1-h,+\infty)}\left(\mathbb{Z}_{p}^{\times}, V\right)\right),
\end{aligned}
$$

where the last map is the connecting map of the following exact sequence:

$$
0 \longrightarrow \mathscr{D}_{\mathrm{alg}}^{I}\left(\mathbb{Z}_{p}^{\times}, V\right) \longrightarrow \mathscr{D}_{\mathrm{alg}}^{I}\left(\mathbb{Z}_{p}^{\times}, B_{\mathrm{max}}^{\varphi=1} \otimes V\right) \longrightarrow \mathscr{D}_{\mathrm{alg}}^{I}\left(\mathbb{Z}_{p}^{\times}, B_{\mathrm{dR}} / B_{\mathrm{dR}}^{+} \otimes V\right) \longrightarrow 0
$$


Recall that

$$
\tilde{\mathscr{D}}_{\text {temp }}\left(\mathbb{Q}_{p}, D(V)\right)=\lim _{\text {temp }}\left(\mathbb{Q}_{p}, D(V(k))\right),
$$

where the projective limit map is given by $\mu \rightarrow(-t x) \mu$. Then Perrin-Riou [9] first proved the following theorem.

THEOREM 5.1 (Perrin-Riou). Assume that $V$ is a crystalline representation, $h \in \mathbb{Z}$ such that $\mathrm{Fil}^{-h} D(V)=D(V)$. If $\mu \in \tilde{\mathscr{D}}_{\mathrm{temp}}\left(\mathbb{Q}_{p}, D(V)\right)^{\Phi=1}$, then $\operatorname{Exp}_{h, V}(\mu)$ restricted to $K_{\infty}$ is in $H^{1}\left(K_{\infty}, \mathscr{D}_{\text {temp }}\left(\mathbb{Z}_{p}^{\times}, V\right)\right)$.

From Section 4 , we know that for $\mu_{\beta} \in \tilde{\mathscr{D}}_{\text {temp }}\left(\mathbb{Q}_{p}, D\left(\mathbb{Q}_{p}(1)\right)\right)^{\Phi=1}$, we could have that $\mathscr{F}_{\text {alg }}\left(\mu_{\beta}\right)$ is not tempered, so the miracle of this theorem is that $\operatorname{Exp}_{h, V}$ sends tempered distribution to tempered distribution (not only algebraic distribution). Then Perrin-Riou gets the following theorem.

THEOREM 5.2 (Perrin-Riou). Assume that $V$ is a crystalline representation, $h \in \mathbb{Z}$ such that $\mathrm{Fil}^{-h} D(V)=D(V)$, for $k \geq 1-h$,

$$
\begin{gathered}
\int_{\mathbb{Z}_{p}^{\times}} x^{k} \operatorname{Exp}_{h, V}(\mu)=\exp _{V(k)}\left((1-\varphi)^{-1}\left(1-p^{-1} \varphi^{-1}\right)\left((k+h-1) ! \int_{\mathbb{Z}_{p}^{\times}} \frac{\mu}{(-t x)^{k}}\right)\right), \\
\int_{a+p^{n} \mathbb{Z}_{p}} x^{k} \operatorname{Exp}_{h, V}(\mu)=(k+h-1) ! \exp _{V(k)}\left(\frac{\varphi^{-n}}{p^{n}}\left(\int_{\mathbb{Z}_{p}} \varepsilon\left(\frac{a x}{p^{n}}\right) \frac{\mu}{(-t x)^{k}}\right)\right), \\
\text { for } n \geq 1, a \in \mathbb{Z}_{p}^{\times} .
\end{gathered}
$$

The significance of this theorem is that for $k \in \mathbb{Z}_{p}$, the left-hand side (hence the righthand side) gives an analytic family of cohomology classes in the sense of Section 3.

The ring $\mathscr{D}_{0}\left(\mathbb{Z}_{p}^{\times}, \mathbb{Q}_{p}\right)$ has an action on both the distribution side $\mathscr{D}_{\text {alg }}^{(-\infty, 1-h]}\left(\mathbb{Q}_{p}, D(V)\right)$ and the cohomology side $H^{1}\left(\mathbb{Q}_{p}, \mathscr{D}_{\text {alg }}^{[h-1, \infty)}\left(\mathbb{Z}_{p}^{\times}, V\right)\right)$. That is, for $\lambda \in \mathscr{D}_{0}\left(\mathbb{Z}_{p}^{\times}, \mathbb{Q}_{p}\right)$ and $\mu \in \mathscr{D}_{\text {alg }}^{(-\infty, 1-h]}\left(\mathbb{Q}_{p}, D(V)\right), \xi \in H^{1}\left(\mathbb{Q}_{p}, \mathscr{D}_{\text {alg }}^{[h-1, \infty)}\left(\mathbb{Z}_{p}^{\times}, V\right)\right)$, then the action $*$ (which is essentially induced by the map $\left.\mathbb{Z}_{p}^{\times} \times \mathbb{Q}_{p} \rightarrow \mathbb{Q}_{p},(x, y) \rightarrow x y\right)$ is defined as

$$
\begin{aligned}
\int_{\mathbb{Q}_{p}} f(x) \lambda * \mu:=\int_{\mathbb{Q}_{p}} \int_{\mathbb{Z}_{p}^{\times}} f(x y) \lambda(x) \mu(y), \\
\int_{\mathbb{Z}_{p}^{\times}} f(x) \lambda * \xi:=\int_{\mathbb{Z}_{p}^{\times}} \int_{\mathbb{Z}_{p}^{\times}} f(x y) \lambda(x) \xi(y) .
\end{aligned}
$$

LEMMA 5.3. (i) The action (5.7) commutes with the action $\Phi$, hence induces an action on $\mathscr{D}_{\text {alg }}^{I}\left(\mathbb{Q}_{p}, D(V)\right)^{\Phi=1}$, and it sends tempered distributions to tempered distributions.

(ii) The action (5.8) commutes with the Galois action, hence it is well defined on $H^{1}\left(\mathbb{Q}_{p}, \mathscr{D}_{\text {alg }}^{I}\left(\mathbb{Z}_{p}^{\times}, V\right)\right)$.

(iii) The map $\operatorname{Exp}_{h, V}$ is sesquilinear with respect to these actions, that is,

$$
\operatorname{Exp}_{h, V}(\lambda * \mu)=\lambda^{\sqrt{ }} * \operatorname{Exp}_{h, V}(\mu),
$$

where $\sqrt{ }$ is induced by $x \rightarrow x^{-1}$ and defined to be

$$
\int_{\mathbb{Z}_{p}^{\times}} f(x) \lambda^{\sqrt{ }}=\int_{\mathbb{Z}_{p}^{\times}} f\left(x^{-1}\right) \lambda(x) .
$$


Proof. These follow from the definitions.

For the "negative" power, Colmez proved the following theorem.

Theorem 5.4 (Colmez). Assume that $V$ is a crystalline representation, $h \in \mathbb{Z}, k \geq h$, then

$$
\exp _{V(-k)}^{*}\left(\int_{\mathbb{Z}_{p}^{\times}} x^{-k} \operatorname{Exp}_{h, V}(\mu)\right)=(-1)^{h-1}\left(1-p^{-1} \varphi^{-1}\right) \int_{\mathbb{Z}} \frac{(t x)^{k}}{(k-h) !} \mu .
$$

REMARK 5.5. Colmez [2] proved Theorem 5.4, for $k \gg 1$; we will prove the statement for $k \geq h$ in another paper [11]; this can also be found in [12]. From his proof, we can get the following theorem.

THEOREM 5.6. Assume that $h \in \mathbb{Z}$ and $\mu \in \tilde{\mathscr{D}}_{\mathrm{temp}}\left(\mathbb{Q}_{p}, D(V)\right)^{\Phi=1}$, for $k \geq h, n \geq 1$, then

$$
\exp _{V(-k)}^{*}\left(\int_{1+p^{n} \mathbb{Z}_{p}} x^{-k} \operatorname{Exp}_{h, V}(\mu)\right)=(-1)^{h-1} \frac{\varphi^{-n}}{p^{n}} \int_{\mathbb{Z}_{p}} \varepsilon\left(\frac{x}{p^{n}}\right) \frac{(t x)^{k}}{(k-h) !} \mu .
$$

Proof. Choose $r \in \mathbb{N}$ large enough such that $F_{r}=\int_{\mathbb{Q}_{p}}\left[\varepsilon^{x}\right]\left(\mu /(-t x)^{r}\right)$ exists. Theorem IV.1.1 in [2] implies that

$$
\delta_{V(-k)} \circ T_{n}\left(F_{r}\right)=\exp _{V(-k)}^{*}\left(\frac{(-1)^{h+r-1}(k-h) !}{(k+r) !} \int_{1+p^{n} \mathbb{Z}_{p}} x^{-k} \operatorname{Exp}_{h, V}(\mu)\right) ;
$$

by using [2, the formula in II.2.1], we get

$$
\exp _{V(-k)}^{*}\left(\int_{1+p^{n} \mathbb{Z}_{p}} x^{-k} \operatorname{Exp}_{h, V}(\mu)\right)=(-1)^{h-1} \cdot p^{-n} \int_{p^{-n} \mathbb{Z}_{p}} \varepsilon(x) \frac{(t x)^{k}}{(k-h) !} \mu,
$$

and the theorem follows from the condition $\Phi(\mu)=\mu$.

The significance of these two theorems is that for $k \gg 1,\left(\exp _{V(-k)}^{*}\right)^{-1}$ gives rise to an analytic family of cohomology. Theorems 5.4 and 5.6 are called explicit reciprocity law.

To get the symmetric form of the explicit reciprocity law, one defines the following pairing:

$$
[\cdot, \cdot]_{D(V)}: \tilde{\mathscr{D}}_{\mathrm{temp}}\left(\mathbb{Q}_{p}, D(V)\right)^{\Phi=1} \times \tilde{\mathscr{D}}_{\mathrm{temp}}\left(\mathbb{Q}_{p}, D\left(V^{*}(1)\right)\right)^{\Phi=1} \longrightarrow \mathscr{D}_{\mathrm{temp}}\left(\mathbb{Z}_{p}^{\times}, \mathbb{Q}_{p}\right)
$$

as

$$
\int_{\mathbb{Z}_{p}^{\times}} f(x)\left[\mu, \mu^{\prime}\right]_{D(V)}=\iint_{\mathbb{Z}_{p}^{\times} \times \mathbb{Z}_{p}^{\times}} f\left(x^{-1} y\right) \mu \otimes \mu^{\prime} .
$$

The pairing in the cohomology side is defined as

$$
\begin{aligned}
(\cdot, \cdot)_{V} & : H^{1}\left(\mathbb{Q}_{p}, \mathscr{D}_{\text {temp }}\left(\mathbb{Z}_{p}^{\times}, V\right)\right) \times H^{1}\left(\mathbb{Q}_{p}, \mathscr{D}_{\text {temp }}\left(\mathbb{Z}_{p}^{\times}, V^{*}(1)\right)\right) \\
& \longrightarrow H^{2}\left(\mathbb{Q}_{p}, \mathscr{D}_{\text {temp }}\left(\mathbb{Z}_{p}^{\times} \times \mathbb{Z}_{p}^{\times}, V \otimes V^{*}(1)\right)\right) \\
& \simeq \mathscr{D}_{\text {temp }}\left(\mathbb{Z}_{p}^{\times}, H^{2}\left(\mathbb{Z}_{p}, \mathbb{Q}_{p}(1)\right)\right) \\
& \simeq \mathscr{D}_{\text {temp }}\left(\mathbb{Z}_{p}^{\times}, \mathbb{Q}_{p}\right) .
\end{aligned}
$$


From Theorems 5.4 and 5.6, we have the following theorem.

THeOREM 5.7 (Perrin-Riou and Colmez). Assume that $V$ is crystalline representation of $G_{\mathbb{Q}_{p}}, \mu \in \tilde{\mathscr{D}}_{\text {temp }}\left(\mathbb{Q}_{p}, D(V)\right)^{\Phi=1}, \mu^{\prime} \in \tilde{\mathscr{D}}_{\text {temp }}\left(\mathbb{Q}_{p}, D\left(V^{*}(1)\right)\right)^{\Phi=1}$, then

$$
\left(\operatorname{Exp}_{h, V}(\mu), \operatorname{Exp}_{1-h, V^{*}(1)}\left(\mu^{\prime}\right)\right)=(-1)^{h}\left[\delta_{-1} * \mu, \mu^{\prime}\right]_{D(V)},
$$

where $\delta_{-1}$ is defined by

$$
\int_{\mathbb{Q}_{p}} f(x) \delta_{-1} * \mu=\int_{\mathbb{Q}_{p}} f(-x) \mu \text {. }
$$

Perrin-Riou proved this theorem for $V=\mathbb{Q}_{p}(1)$ and Colmez proved it for general crystalline representation.

Moreover, as Iwasawa modules, those pairings have the following properties.

Proposition 5.8. (i) For $\mu \in \tilde{\mathscr{D}}_{\text {temp }}\left(\mathbb{Q}_{p}, D(V)\right)^{\Phi=1}$ and $\mu^{\prime} \in \tilde{\mathscr{D}}_{\text {temp }}\left(\mathbb{Q}_{p}, D\left(V^{*}(1)\right)\right)^{\Phi=1}$, the integral

$$
\int_{\mathbb{Z}_{p}^{\times}} x^{i}\left[\mu, \mu^{\prime}\right]_{D(V)}=\left[\int_{\mathbb{Z}_{p}^{\times}} x^{-i} \mu, \int_{\mathbb{Z}_{p}^{\times}} x^{i} \mu^{\prime}\right]_{D(V)},
$$

where the last pairing is defined in Section 3.

(ii) For $\xi \in H^{1}\left(\mathbb{Q}_{p}, \mathscr{D}_{\text {temp }}\left(\mathbb{Z}_{p}^{\times}, V\right)\right)$ and $\xi^{\prime} \in H^{1}\left(\mathbb{Q}_{p}, \mathscr{D}_{\text {temp }}\left(\mathbb{Z}_{p}^{\times}, V^{*}(1)\right)\right)$, the integral

$$
\int_{\mathbb{Z}_{p}^{\times}} x^{i}\left(\xi, \xi^{\prime}\right)_{V}=\int_{\mathbb{Z}_{p}^{\times}} x^{i} \xi \cup \int_{\mathbb{Z}_{p}^{\times}} x^{-i} \xi^{\prime}
$$

where the cup product is given by

$$
H^{1}\left(\mathbb{Q}_{p}, V(i)\right) \cup H^{1}\left(\mathbb{Q}_{p}, V^{*}(1-i)\right) \longrightarrow H^{2}\left(\mathbb{Q}_{p}, \mathbb{Q}_{p}(1)\right) \simeq \mathbb{Q}_{p}
$$

(iii) $[\cdot, \cdot]_{D(V)}$ is sesquilinear for the first variable and linear for the second variable, that is,

$$
\begin{aligned}
{\left[\delta * \mu, \mu^{\prime}\right]_{D(V)} } & =\delta^{\sqrt{ }} *\left[\mu, \mu^{\prime}\right]_{D(V)} \\
{\left[\mu, \delta * \mu^{\prime}\right]_{D(V)} } & =\delta *\left[\mu, \mu^{\prime}\right]_{D(V)}
\end{aligned}
$$

(iv) $(\cdot, \cdot)_{V}$ is linear for the first variable and sesquilinear for the second variable, that is,

$$
\begin{aligned}
& \left(\delta * \xi, \xi^{\prime}\right)_{V}=\delta *\left(\xi, \xi^{\prime}\right)_{V}, \\
& \left(\xi, \delta * \xi^{\prime}\right)_{V}=\delta^{\sqrt{ }} *\left(\xi, \xi^{\prime}\right)_{V} .
\end{aligned}
$$


Proof. (i) and (ii) are just from definitions, which can also be found in Colmez [2]. For (iii), we have

$$
\begin{aligned}
\int_{\mathbb{Z}_{p}^{\times}} x^{i}\left[\delta * \mu, \mu^{\prime}\right]_{D(V)} & =\left[\int_{\mathbb{Z}_{p}^{\times}} x^{-i}(\delta * \mu), \int_{\mathbb{Z}_{p}^{\times}} x^{i} \mu^{\prime}\right]_{D(V)} \\
& =\left[\int_{\mathbb{Z}_{p}^{\times}} \int_{\mathbb{Z}_{p}^{\times}} x^{-i} y^{-i} \delta(y) \mu(x), \int_{\mathbb{Z}_{p}^{\times}} x^{i} \mu^{\prime}\right]_{D(V)} \\
& =\int_{\mathbb{Z}_{p}^{\times}} y^{-i} \delta(y)\left[\int_{\mathbb{Z}_{p}^{\times}} x^{-i} \mu, \int_{\mathbb{Z}_{p}^{\times}} x^{i} \mu^{\prime}\right]_{D(V)} \\
& =\int_{\mathbb{Z}_{p}^{\times}} x^{i} \delta^{\sqrt{ }} *\left[\mu, \mu^{\prime}\right]_{D(V)},
\end{aligned}
$$

and (iv) is similar to (iii).

6. Iwasawa's explicit reciprocity law. Recall that $K_{n}=\mathbb{Q}_{p}\left(\zeta_{p^{n}}\right)$ and let $D=(1+$ $T)(d / d T)$. For $\beta \in \lim _{K_{n}}^{\times}$, let $g_{\beta}(T) \in \mathbb{Z}_{p}[[T]]$ denote the Coleman power series.

Theorem 6.1 (Iwasawa). Let $\alpha_{n}, \beta_{n} \in O_{K_{n}}$ such that $\alpha_{n} \equiv 1\left(\omega_{n}\right)$ and $\beta_{n} \in K_{n}^{\times}$sits in a norm coherence sequence $\beta=\left(\beta_{n}\right)_{n}$, let $g_{\beta}$ denote the Coleman power series corresponding to $\beta$, and define

$$
\begin{gathered}
\left(\alpha_{n}, \beta_{n}\right)_{n}=\left(\alpha_{n}^{1 / p^{n}}\right)^{\sigma_{\beta n}-1}, \\
{\left[\alpha_{n}, \beta_{n}\right]_{n}=p^{-n} \operatorname{Tr}_{K_{n} / \mathbb{Q}_{p}}\left(\log \alpha_{n} D \log g_{\beta}\left(\omega_{n}\right)\right)\left(\bmod p^{n}\right) .}
\end{gathered}
$$

Then

$$
\left(\alpha_{n}, \beta_{n}\right)_{n}=\zeta_{p^{n}}^{\left[\alpha_{n}, \beta_{n}\right]_{n}}
$$

where $\omega_{n}=\zeta_{p^{n}}-1$.

In the following, we will show that Perrin-Riou-Colmez explicit reciprocity law, Theorem 5.2, implies Iwasawa's explicit reciprocity law.

Recall that we have the Bloch-Kato exponential map $\exp _{K_{n}, V}:\left(B_{\mathrm{dR}} \otimes V\right)^{G_{K_{n}}} \rightarrow H^{1}\left(K_{n}, V\right)$.

Let $V=\mathbb{Q}_{p}(1)$ and let $U_{n}$ denote the principal units of $O_{K_{n}}$. To an element of $\lim _{U_{n}}$, we will associate an element in $\tilde{D}_{1}\left(\mathbb{Q}_{p}, D(V)\right)^{\Phi=1}$. To an element in $\lim K_{n}^{\times}$, we will associate an element in $H^{1}\left(\mathbb{Q}_{p}, \mathscr{D}_{0}\left(\mathbb{Z}_{p}^{\times}, V\right)\right)$.

For $\beta \in \lim _{n} U_{n}$, define $\mu_{\beta} \in \mathscr{D}_{\text {alg }}^{+}\left(\mathbb{Z}_{p}, D(V)\right)$ as

$$
\int_{\mathbb{Z}_{p}}(1+T)^{x} \mu_{\beta}=\log g_{\beta}(T) \otimes \frac{e}{t},
$$

and extend it to $\mathbb{Q}_{p}$ by defining

$$
\int_{p^{-n} \mathbb{Z}_{p}} f(x) \mu_{\beta}=p^{n} \int_{\mathbb{Z}_{p}} f\left(p^{-n} x\right) \mu_{\beta}=\varphi^{-n} \int_{\mathbb{Z}_{p}} f\left(p^{-n} x\right) \mu_{\beta} .
$$


Then

$$
\begin{aligned}
\int_{\mathbb{Q}_{p}} f(x) \Phi \mu_{\beta} & =\varphi\left(\int_{\mathbb{Q}_{p}} f(p x) \mu_{\beta}\right) \\
& =\frac{1}{p} \cdot \int_{\mathbb{Q}_{p}} f(p x) \mu_{\beta} \\
& =\frac{1}{p} \cdot p \int_{\mathbb{Q}_{p}} f(x) \mu_{\beta} \\
& =\int_{\mathbb{Q}_{p}} f(x) \mu_{\beta},
\end{aligned}
$$

hence $\Phi \mu_{\beta}=\mu_{\beta}$. By Proposition 4.2, $\mu_{\beta}$ is 1-admissible. Note that Coleman power series has the property $g_{\beta_{1} \beta_{2}}=g_{\beta_{1}} \cdot g_{\beta_{2}}$. So we get a map

$$
\underset{\lim }{\longleftarrow} U_{n} \longrightarrow \mathscr{D}_{1}\left(\mathbb{Q}_{p}, D\left(\mathbb{Q}_{p}(1)\right)\right)^{\Phi=1} \text {. }
$$

On the other hand, for $\beta \in \lim _{n}^{\times}, \beta=\left(\beta_{n}\right)$, then $\beta_{n}$ gives $\gamma_{\beta_{n}} \in H^{1}\left(K_{n}, \mathbb{Z}_{p}(1)\right)$ defined by Kummer map. By using Colmez's theorem in Section 5 , we get an element $\hat{\beta}(\tau):=$ $\left\lfloor\gamma_{\beta_{n}}(\tau) \in \lim ^{1} H^{1}\left(K_{n}, \mathbb{Z}_{p}(1)\right) \cong H^{1}\left(\mathbb{Q}_{p}, \mathscr{D}_{0}\left(\mathbb{Z}_{p}^{\times}, \mathbb{Z}_{p}(1)\right)\right)\right.$. Hence, we have a map

$$
\begin{aligned}
\lim _{n} K_{n}^{\times} & \longrightarrow H^{1}\left(\mathbb{Q}_{p}, \mathscr{D}_{0}\left(\mathbb{Z}_{p}^{\times}, \mathbb{Z}_{p}(1)\right)\right), \\
\beta & \longrightarrow \hat{\beta},
\end{aligned}
$$

which has the property $\widehat{\beta_{1} \cdot \beta_{2}}=\widehat{\beta_{1}}+\widehat{\beta_{2}}$.

We can also state this map by using integral, namely, for $\beta \in \varliminf_{\underline{m}} K_{n}^{\times}, \hat{\beta} \in H^{1}\left(\mathbb{Q}_{p}, \mathscr{D}_{0}\left(\mathbb{Z}_{p}^{\times}\right.\right.$, $\left.\left.\mathbb{Z}_{p}(1)\right)\right)$ is the element such that

$$
\int_{1+p^{n} \mathbb{Z}_{p}} \hat{\beta}=\gamma_{\beta_{n}}, \quad \int_{a+p^{n} \mathbb{Z}_{p}} \hat{\beta}=\gamma_{\sigma_{a}\left(\beta_{n}\right)} .
$$

Especially, for $\beta \in \underline{\lim } U_{n}$, we have

$$
\mu_{\beta} \in \mathscr{D}_{1}\left(\mathbb{Q}_{p}, D\left(\mathbb{Q}_{p}(1)\right)\right)^{\Phi=1}, \quad \hat{\beta} \in H^{1}\left(\mathbb{Q}_{p}, \mathscr{D}_{0}\left(\mathbb{Z}_{p}^{\times}, \mathbb{Z}_{p}(1)\right)\right) .
$$

The element $\left(p, 1-\zeta_{p}, 1-\zeta_{p^{2}}, \ldots\right) \in \varliminf_{\lim } K_{n}^{\times}$gives an element in $H^{1}\left(\mathbb{Q}_{p}, \mathscr{D}_{0}\left(\mathbb{Z}_{p}^{\times}, \mathbb{Z}_{p}(1)\right)\right)$, we denote it by $\hat{p}$. Fix $a, b \in \mathbb{Z}_{p}^{\times}$such that $a \equiv b(\bmod p), a \neq b$. For example, we can take $a=1$ and $b=1+p$. Then the element $\left(\ldots,\left(\zeta_{p^{n}}^{a}-1\right) /\left(\zeta_{p^{n}}^{b}-1\right), \ldots\right) \in \underline{\lim } U_{n}$, hence gives a distribution, which we denote by $\mu_{a b}$. Recall that $\delta_{a} \in \mathscr{D}_{0}\left(\mathbb{Z}_{p}^{\times}, \mathbb{Z}_{p}\right)$ is defined to be the Dirac measure

$$
\int_{\mathbb{Z}_{p}^{\times}} f(x) \delta_{a}=f(a)
$$

Let $\delta_{a b}=\delta_{a}-\delta_{b} \in \mathscr{D}_{0}\left(\mathbb{Z}_{p}^{\times}, \mathbb{Z}_{p}\right)$. The following lemma describes the relationship between $\mu_{\beta}$ and $\hat{\beta}, \mu_{a b}$ and $\hat{p}$.

LEMMA 6.2. (i) There is a homomorphism $\mu: \varliminf_{n} U_{n} \rightarrow \mathscr{D}_{1}\left(\mathbb{Q}_{p}, D\left(\mathbb{Q}_{p}(1)\right)\right)^{\Phi=1}$, which sends $\beta$ to $\mu_{\beta}$. 
(ii) There is a map $\xi: \lim _{n}^{\times} \rightarrow H^{1}\left(\mathbb{Q}_{p}, \mathscr{D}_{0}\left(\mathbb{Z}_{p}^{\times}, \mathbb{Z}_{p}(1)\right)\right)$, which sends $\beta$ to $\hat{\beta}$.

(iii) For $\beta \in \underset{\lim }{U_{n}}, \operatorname{Exp}_{1, \mathbb{Q}_{p}(1)}\left(\mu_{\beta}\right)=\hat{\beta}$.

(iv) For $a, b \in \mathbb{Z}_{p}^{\times}, a \equiv b(\bmod p), a \neq b$,

$$
\operatorname{Exp}_{1, \mathbb{Q}_{p}(1)}\left(\mu_{a b}\right)=\delta_{a b}^{\sqrt{ }} * \hat{p} .
$$

Proof. We have already proved (i) and (ii). For (iii), by Theorem 5.2,

$$
\begin{aligned}
\int_{a+p^{n} \mathbb{Z}_{p}} \operatorname{Exp}_{1, \mathbb{Q}_{p}(1)}\left(\mu_{\beta}\right) & =\exp _{\mathbb{Q}_{p}(1)}\left(\frac{\varphi^{-n}}{p^{n}} \int_{\mathbb{Z}_{p}} \varepsilon\left(\frac{a x}{p^{n}}\right) \mu_{\beta}\right) \\
& =\exp _{\mathbb{Q}_{p}(1)}\left(\frac{\varphi^{-n}}{p^{n}} \frac{\log g_{\beta}\left(\sigma_{a}\left(\omega_{n}\right)\right)}{t}\right) \\
& =\exp _{\mathbb{Q}_{p}(1)}\left(\frac{\log \sigma_{a}\left(\beta_{n}\right)}{t}\right) \\
& =\gamma_{\sigma_{a}\left(\beta_{n}\right)} \\
& =\int_{a+p^{n} \mathbb{Z}_{p}} \hat{\beta} .
\end{aligned}
$$

(iv) Let $\beta=\left(\ldots,\left(\zeta_{p^{n}}^{a}-1\right) /\left(\zeta_{p^{n}}^{b}-1\right), \ldots\right)$. From (iii), we have $\operatorname{Exp}_{1, \mathbb{Q}_{p}(1)}\left(\mu_{a b}\right)=\hat{\beta}$. So we only need to look at the relation between $\hat{\beta}$ and $\hat{p}$. Let $p_{a}=\left(\ldots, \zeta_{p^{n}}^{a}-1, \ldots\right)$, then by (ii) we have $\hat{\beta}=\widehat{p_{a}}-\widehat{p_{b}}$.

The integral

$$
\begin{aligned}
\int_{1+p^{n} \mathbb{Z}_{p}} \delta_{a^{-1}} * \hat{p} & =\int_{\mathbb{Z}_{p}^{\times}} 1_{1+p^{n} \mathbb{Z}_{p}}(x y) \delta_{a^{-1}}(x) \hat{p}(y) \\
& =\int_{\mathbb{Z}_{p}^{\times}} 1_{1+p^{n} \mathbb{Z}_{p}}\left(a^{-1} y\right) \hat{p}(y) \\
& =\int_{a+p^{n} \mathbb{Z}_{p}} \hat{p}(y) \\
& =\int_{1+p^{n} \mathbb{Z}_{p}} \widehat{\sigma_{a}(p)} \\
& =\int_{1+p^{n} \mathbb{Z}_{p}} \widehat{p_{a}},
\end{aligned}
$$

hence $\widehat{p_{a}}=\delta_{a^{-1}} * \hat{p}$. And we have $\hat{\beta}=\left(\delta_{a^{-1}}-\delta_{b^{-1}}\right) * \hat{p}=\delta_{a b}^{\sqrt{ }} * \hat{p}$. Hence, the lemma follows.

The relation $\operatorname{Exp}_{1, \mathbb{Q}_{p}(1)}\left(\mu_{a b}\right)=\delta_{a b}^{\vee} * \hat{p}$ can make us extend Perrin-Riou exponential map to some elements with denominator $\delta_{a b}$ and we can define

$$
\operatorname{Exp}_{1, \mathbb{Q} p(1)}\left(\frac{\mu_{a b}}{\delta_{a b}}\right)=\hat{p}
$$

This relation is significant in the sense that Bloch-Kato map $\exp _{\mathbb{Q}_{p}(1)}$ cannot have $\gamma_{p}$ as the image, but Perrin-Riou map $\operatorname{Exp}_{1, \mathbb{Q}_{p}(1)}$ can have $\hat{p}$ as the image. If we use Colmez's $\operatorname{logarithm}$ to explain this, this will correspond to $\log (\hat{p}) \neq 0$, but $\log (p)=0$. Another remark is that $\mu_{a b} / \delta_{a b}$ does not depend on the choice of $a, b$ subject to the condition 
$a \equiv b \bmod p$. This can be seen as if we have $c \equiv d \bmod p$, then the Amice transformation of $\delta_{a b} * \mu_{c d}$ is given by $\int_{\mathbb{Z}_{p}}(1+T)^{x} \delta_{a b} * \mu_{c d}=\log \left(\left((1+T)^{a c}-1\right)\left((1+T)^{b d}-1\right) /((1+\right.$ $\left.\left.T)^{b c}-1\right)\left((1+T)^{a d}-1\right)\right)$ which is the same as the Amice transformation of $\delta_{c d} * \mu_{a b}$. Hence, $\delta_{a b} * \mu_{c d}=\delta_{c d} * \mu_{a b}$. We denote this pseudomeasure $\mu_{a b} / \delta_{a b}$ by $\mu_{p}$. For a crystalline representation $W$ such that there is a Galois inclusion $\mathbb{Q}_{p}(1) \subset W$, we have the following theorem.

THEOREM 6.3. For $W$ above, the $\operatorname{map} \operatorname{Exp}_{1, V}(\mu)$ can be extended to the set including $\mu_{p}$ by using the inclusion $H^{1}\left(\mathbb{Q}_{p}, \mathscr{D}_{0}\left(\mathbb{Z}_{p}^{\times}, \mathbb{Q}_{p}(1)\right)\right) \hookrightarrow H^{1}\left(\mathbb{Q}_{p}, \mathscr{D}_{0}\left(\mathbb{Z}_{p}^{\times}, W\right)\right)$. For $\mu \in\left\langle\mu_{p}\right\rangle \oplus$ $\tilde{\mathscr{D}}_{\text {temp }}\left(\mathbb{Q}_{p}, D(W)\right)^{\Phi=1}, \mu^{\prime} \in \tilde{\mathscr{D}}\left(\mathbb{Q}_{p}, D\left(W^{*}(1)\right)\right)^{\Phi=1}$,

$$
\left(\operatorname{Exp}_{1, W}(\mu), \operatorname{Exp}_{0, W^{*}(1)}\left(\mu^{\prime}\right)\right)_{W}=-\left[\delta_{-1} * \mu, \mu^{\prime}\right]_{D(W)} .
$$

Proof. We only need to show that for $\mu=\mu_{p}$, this will follow from the definition, the sesquilinear property of the exponential map, and the pairings

$$
\begin{aligned}
& \left(\operatorname{Exp}_{1, W}\left(\mu_{a b}\right), \operatorname{Exp}_{W^{*}(1), 0}\left(\mu^{\prime}\right)\right)=-\left[\delta_{-1} * \mu_{a b}, \mu^{\prime}\right],
\end{aligned}
$$

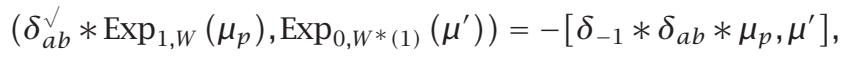

$$
\begin{aligned}
& \delta_{a b}^{\sqrt{ }} *\left(\operatorname{Exp}_{1, W}\left(\mu_{p}\right), \operatorname{Exp}_{0, W *(1)}\left(\mu^{\prime}\right)\right)=-\delta_{a b}^{\sqrt{ }} *\left[\delta_{-1} * \mu_{p}, \mu^{\prime}\right],
\end{aligned}
$$

since the convolution in $\mathscr{D}_{0}\left(\mathbb{Z}_{p}^{\times}, \mathbb{Q}_{p}\right)$ has cancellation law. This implies that

$$
\left(\operatorname{Exp}_{1, W}\left(\mu_{p}\right), \operatorname{Exp}_{0, W^{*}(1)}\left(\mu^{\prime}\right)\right)=-\left[\delta_{-1} * \mu_{p}, \mu^{\prime}\right] .
$$

(This can also be seen from that if $\delta_{a b} * \mu=0$, then $\int y^{k} \delta_{a b} * \mu=0$, hence $\int y^{k} \mu=0$, hence $\mu=0$.)

Now, we use Perrin-Riou and Colmez explicit reciprocity law to prove Iwasawa's explicit reciprocity law. Assume that $\alpha_{n}, \beta_{n} \in O_{K_{n}} \backslash\{0\}, \beta_{n}$ sits in a norm coherence sequence. Then $\beta_{n}=u_{n} \cdot \beta_{n}^{\prime} \cdot \omega_{n}^{j}$ for $u_{n}$ a $(p-1)$ th root of unity, $\beta_{n}^{\prime} \equiv 1\left(\bmod \omega_{n}\right)$, $j \geq 0$. We know that the $u_{n}$ will give $\left(\alpha_{n}, u_{n}\right)_{n}=1$ and $\sigma_{u_{n}}=1$. So we will consider the case $\beta_{n} \equiv 1\left(\bmod \omega_{n}\right)$ and the case $\beta_{n}=\omega_{n}$ separately. For $\beta$ with $\beta_{n} \equiv 1\left(\bmod \omega_{n}\right)$, we have $\mu_{\beta} \in \mathscr{D}_{1}\left(\mathbb{Q}_{p}, D\left(\mathbb{Q}_{p}(1)\right)\right)^{\Phi=1}$. Then $(-t x) \mu_{\beta} \in \mathscr{D}_{1}\left(\mathbb{Q}_{p}, \mathbb{Q}_{p}\right)^{\Phi=1}$.

LEMMA 6.4. (i) For $\alpha_{n} \in O_{K_{n}} \backslash[0]$, $\exp _{K_{n}, \mathbb{Q}_{p}(1)}\left(\left(\log \left(\alpha_{n}\right) / t\right) \otimes e\right)=\gamma_{\alpha_{n}}$.

(ii) For all $\mu \in \tilde{\mathscr{D}}_{\text {temp }}\left(\mathbb{Q}_{p}, D\left(\mathbb{Q}_{p}(1)\right)\right)^{\Phi=1}, \operatorname{Exp}_{1, \mathbb{Q}_{p}}(1)(\mu)=x \operatorname{Exp}_{0, \mathbb{Q}_{p}}(-t x \mu)$

(iii) If $\beta$ has the property $\beta_{n} \equiv 1\left(\bmod \omega_{n}\right), \operatorname{Exp}_{0, \mathbb{Q} p}\left(-t x \mu_{\beta}\right)=\hat{\beta} / x$.

(iv) The integral $\int_{\mathbb{Z}_{p}} \varepsilon\left(x / p^{n}\right) x \mu_{p}=\zeta_{p^{n}} / \omega_{n}$.

Proof. (i) Since $\left(\log \left[\widetilde{\alpha_{n}}\right] / t\right) \otimes e \in B_{\text {crys }}^{\varphi=1}(1)$ is a lifting of $\left(\log \left(\alpha_{n}\right) / t\right) \otimes e$, by the definition of exponential map and the Kummer class, we see (i). From [2] we know that

$$
\begin{aligned}
& \int_{1+p^{n} \mathbb{Z}_{p}} x^{k} \operatorname{Exp}_{1, \mathbb{Q}_{p}(1)}(\mu)=k ! \exp _{\mathbb{Q}_{p}(k+1)}\left(\frac{\varphi^{-n}}{p^{n}}\left(\int_{\mathbb{Z}_{p}} \frac{\mu}{(-t x)^{k}}\right)\right), \\
& \int_{1+p^{n} \mathbb{Z}_{p}} x^{k+1} \operatorname{Exp}_{0, \mathbb{Q}_{p}}(-t x \mu)=k ! \exp _{\mathbb{Q}_{p}(k+1)}\left(\frac{\varphi^{-n}}{p^{n}}\left(\int_{\mathbb{Z}_{p}} \frac{\mu}{(-t x)^{k}}\right)\right),
\end{aligned}
$$

then assertion (ii) follows. 
(iii) follows from (ii) and Lemma 6.2(iii).

(iv) Since $\mu_{a b}=\delta_{a b} * \mu_{p}$ and this relation does not depend on the choices of $a$ and $b$, we can take $a=1+p^{n}, b=1$,

$$
\begin{aligned}
\int_{\mathbb{Z}_{p}} \varepsilon\left(\frac{x}{p^{n}}\right) x \mu_{a b} & =\int_{\mathbb{Z}_{p}} \varepsilon\left(\frac{a x}{p^{n}}\right) a x \mu_{p}-\int_{\mathbb{Z}_{p}} \varepsilon\left(\frac{b x}{p^{n}}\right) b x \mu_{p} \\
& =\int_{\mathbb{Z}_{p}} \varepsilon\left(\frac{x}{p^{n}}\right)\left(\left(1+p^{n}\right) x \mu_{p}-x \mu_{p}\right) \\
& =p^{n} \int_{\mathbb{Z}_{p}} \varepsilon\left(\frac{x}{p^{n}}\right) x \mu_{p}
\end{aligned}
$$

since $\mu_{a b}$ corresponds to the power series $\left((1+T)^{1+p^{n}}-1\right) / T$, hence

$$
\begin{aligned}
\int_{\mathbb{Z} p} \varepsilon\left(\frac{x}{p^{n}}\right) x \mu_{a b} & =\left.(1+T) \frac{T}{(1+T)^{1+p^{n}}-1} \frac{d}{d T}\left(\frac{(1+T)^{1+p^{n}}-1}{T}\right)\right|_{T=\omega_{n}} \\
& =\zeta_{p^{n}} \cdot \frac{\omega_{n}}{\omega_{n}} p^{n} \cdot \frac{1}{\omega_{n}} \\
& =p^{n} \frac{\zeta_{p^{n}}}{\omega_{n}}
\end{aligned}
$$

and this completes the proof of this lemma.

Now, we come to the proof of Iwasawa's explicit reciprocity law in two cases.

CASE 1. Assume that $\beta_{n} \equiv 1\left(\bmod \omega_{n}\right)$ sits in the norm coherence sequence $\beta$. Take $V=\mathbb{Q}_{p}(1), h=1, k=1$, and $\mu=\mu_{\beta} \in \tilde{\mathscr{D}}_{\text {temp }}\left(\mathbb{Q}_{p}, D(V)\right)^{\Phi=1}$, and using Theorem 5.6, we have

$$
\exp _{\mathbb{Q}_{p}}^{*}\left(\int_{1+p^{n} \mathbb{Z}_{p}} x^{-1} \hat{\beta}\right)=\frac{\varphi^{-n}}{p^{n}}\left(\int_{\mathbb{Z}_{p}} \varepsilon\left(\frac{x}{p^{n}}\right)(t x) \mu_{\beta}\right),
$$

that is,

$$
\exp _{\mathbb{Q}_{p}}^{*}\left(\int_{1+p^{n} \mathbb{Z}_{p}} x^{-1} \hat{\beta}\right)=\frac{1}{p^{n}} D \log g_{\beta}\left(\omega_{n}\right) .
$$

We already know that

$$
\exp _{\mathbb{Q}_{p}(1)}\left(\frac{\log \alpha_{n}}{t} \otimes e\right)=\gamma_{\alpha_{n}}
$$

From the definition of the Hilbert symbol, we have

$$
\left(\alpha_{n}, \beta_{n}\right)=\zeta_{p^{n}}^{\left(\gamma_{\alpha_{n}}, \gamma_{\beta n}\right)}
$$


From the definition of the dual exponential map, we have

$$
\begin{aligned}
\left(\gamma_{\alpha_{n}}, \gamma_{\beta_{n}}\right) & =\left(\gamma_{\alpha_{n}}, \int_{1+p^{n} \mathbb{Z}_{p}} \hat{\beta}\right) \\
& \equiv\left(\gamma_{\alpha_{n}}, \int_{1+p^{n} \mathbb{Z}_{p}} x^{-1} \hat{\beta}\right) \\
& =\left[\alpha_{n}, \beta_{n}\right] .
\end{aligned}
$$

Hence Iwasawa's explicit reciprocity law follows.

CASE 2. For $\beta_{n}=\omega_{n}, g_{\beta}(T)=T$. Using the sesquilinear property of $\operatorname{Exp}_{h, V}$, we see that

$$
\exp _{\mathbb{Q}_{p}}^{*}\left(\int_{1+p^{n} \mathbb{Z} p} x^{-1} \hat{p}\right)=\frac{\varphi^{-n}}{p^{n}}\left(\int_{\mathbb{Z}_{p}} \varepsilon\left(\frac{x}{p^{n}}\right)(t x) \mu_{p}\right),
$$

that is,

$$
\exp _{\mathbb{Q}_{p}}^{*}\left(\int_{1+p^{n} \mathbb{Z}_{p}} \hat{p}\right)=\frac{1}{p^{n}} D \log (T)\left(\omega_{n}\right) \quad \text { by Lemma 6.4(iv), }
$$

combined with

$$
\exp _{\mathbb{Q}_{p}(1)}\left(\frac{\log \alpha_{n}}{t} \otimes e\right)=\gamma_{\alpha_{n}}
$$

then Iwasawa's explicit reciprocity law follows as in Case 1.

REMARK 6.5. Lemma 6.4(iv) can be interpreted as a completion of the theory of Coleman power series. Namely, $\mu_{p}$ is the distribution whose Amice transformation is $\log (T)$ in the sense that $x \mu_{p}$ corresponds to $D \log (T)$.

7. A trivial zero problem. Recall that $K_{n}=\mathbb{Q}_{p}\left(\zeta_{p^{n}}\right)$ and $u=\underline{\lim } O_{K_{n}}^{\times}$. For $\beta \in \mathcal{U}$, we have a 1-admissible distribution $\mu_{\beta} \in \mathscr{D}_{1}\left(\mathbb{Q}_{p}, \mathbb{Q}_{p}[-1]\right)^{\Phi=1}$. Consider the integral

$$
\psi_{k}(\beta)=\int_{\mathbb{Z}_{p}^{\times}} x^{k} \mu_{\beta},
$$

then

$$
\begin{aligned}
\psi_{k}(\beta) & =\int_{\mathbb{Z}_{p}} x^{k} \mu_{\beta}-\int_{p \mathbb{Z}_{p}} x^{k} \mu_{\beta} \\
& =\int_{\mathbb{Z}_{p}} x^{k} \mu_{\beta}-\int_{\mathbb{Q}_{p}} 1_{p \mathbb{Z}_{p}} x^{k} \mu_{\beta} \\
& =\int_{\mathbb{Z}_{p}} x^{k} \mu_{\beta}-\int_{\mathbb{Q}_{p}} 1_{p \mathbb{Z}_{p}} x^{k} \Phi \mu_{\beta} \\
& =\int_{\mathbb{Z}_{p}} x^{k} \mu_{\beta}-\varphi\left(\int_{\mathbb{Q}_{p}} 1_{p \mathbb{Z}_{p}}(p x)(p x)^{k} \mu_{\beta}\right) \\
& =\left(1-p^{k-1}\right) \int_{\mathbb{Z}_{p}} x^{k} \mu_{\beta} .
\end{aligned}
$$


The Euler factor $1-p^{k-1}$ forces $\psi_{1}=0$ at $k=1$. Since $1-p^{k-1}$ is not an analytic function of $k$, hence we cannot take the derivative directly. But $\psi_{k}$ is an analytic function of $k$, then $d \psi_{k} /\left.d k\right|_{k=1}$ must exist. Glenn Stevens predicted that the derivative of $\psi_{k}$ at 1 will give the Kummer class $\gamma_{p}$. Based on the previous sections, now we can prove that this is true.

LEMMA 7.1. The integral $\int_{\mathbb{Z}_{p}^{\times}} \mu_{a b}=(1-1 / p) \log (a / b)$.

Proof. Since we have

$$
\int_{\mathbb{Z}_{p}^{\times}}(1+T)^{x} \mu_{a b}=\widetilde{\log g}(T),
$$

with $g(T)=\left((1+T)^{a}-1\right) /\left((1+T)^{b}-1\right)$, hence $g(0)=a / b$, and

$$
\int_{\mathbb{Z}_{p}^{\times}} \mu_{a b}=\left.\widetilde{\log g}(T)\right|_{T=0}=\left(1-\frac{1}{p}\right) \log \frac{a}{b} .
$$

LEMMA 7.2. Assume that $\mu \in \mathscr{D}_{\text {temp }}\left(\mathbb{Z}_{p}^{\times}, \mathbb{Q}_{p}\right)$ such that $\int_{\mathbb{Z}_{p}^{\times}} \mu=0$, then

$$
\int_{\mathbb{Z}_{p}^{\times}} \frac{\mu}{\delta_{a b}}=\frac{1}{\log (a / b)} \cdot \lim _{s \rightarrow 0} \frac{1}{s} \int_{\mathbb{Z}_{p}^{\times}}\langle x\rangle^{s} \mu .
$$

Proof. Let $\nu=\mu / \delta_{a b}$ and $\mu=\delta_{a b} * v$,

$$
\begin{aligned}
\int_{\mathbb{Z}_{p}^{\times}}\langle x\rangle^{s} \mu & =\int_{\mathbb{Z}_{p}^{\times}}\langle x\rangle^{s} \delta_{a b} * v \\
& =\int_{\mathbb{Z}_{p}^{\times}}\langle x y\rangle^{s} \delta_{a b}(y) v(x) \\
& =\left(\langle a\rangle^{s}-\langle b\rangle^{s}\right) \int_{\mathbb{Z}_{p}^{\times}}\langle x\rangle^{s} v,
\end{aligned}
$$

hence

$$
\begin{aligned}
\int_{\mathbb{Z}_{\vec{p}}^{\times}} v & =\lim _{s \rightarrow 0} \int_{\mathbb{Z}_{\vec{p}}^{\times}}\langle x\rangle^{s} v \\
& =\lim _{s \rightarrow 0} \frac{1}{\langle a\rangle^{s}-\langle b\rangle^{s}} \int_{\mathbb{Z}_{p}^{\times}}\langle x\rangle^{s} \mu \\
& =\frac{1}{\log (a / b)} \cdot \lim _{s \rightarrow 0} \frac{1}{s} \int_{\mathbb{Z}_{p}^{\times}}\langle x\rangle^{s} \mu .
\end{aligned}
$$

Let $\kappa_{r}: \mathcal{U} \rightarrow H^{1}\left(\mathbb{Q}_{p}, \mathbb{Q}_{p}(1-r)\right)$ be given by $\kappa_{r}(\beta)=\int_{\mathbb{Z}_{p}^{\times}} x^{-r} \hat{\beta}$.

LEMMA 7.3. The following diagram is commutative for $r \geq 1$ :

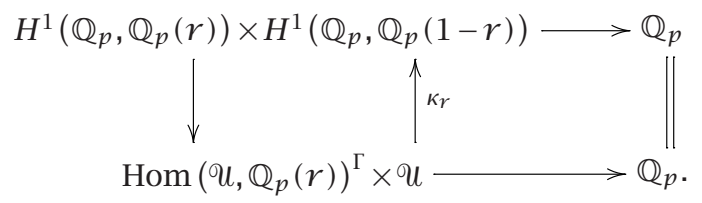


Proof. For $\xi \in H^{1}\left(\mathbb{Q}_{p}, \mathbb{Z}_{p}(r)\right), \beta=\left(\beta_{n}\right)_{n} \in \mathcal{U}$, let $\xi_{n}=\operatorname{res}(\xi) \in H^{1}\left(K_{n}, \mathbb{Z}_{p}(r)\right)$ be the restriction, $\xi_{\infty} \in H^{1}\left(K_{\infty}, \mathbb{Z}_{p}(r)\right) \cong \operatorname{Hom}_{\Gamma}\left(U, \mathbb{Z}_{p}(r)\right)$, then

$$
\xi_{\infty}(\beta)=\lim _{\leftarrow} \xi_{n}\left(\beta_{n}\right)
$$

Since

$$
\begin{gathered}
\int_{1+p^{n} \mathbb{Z}_{p}} x^{-r} \hat{\beta} \equiv \int_{1+p^{n} \mathbb{Z}_{p}} \hat{\beta}\left(\bmod p^{n}\right), \\
\frac{\mathbb{Z}_{p}(1-r)}{p^{n} \mathbb{Z}_{p}} \cong \frac{\mathbb{Z}_{p}}{p^{n} \mathbb{Z}_{p}} \text { as } G_{K_{n}} \text { modules },
\end{gathered}
$$

from the definition of Hilbert symbol we have $\xi_{n}\left(\beta_{n}\right) \equiv\left(\xi_{n}, \int_{1+p^{n} \mathbb{Z}_{p}} x^{-r} \hat{\beta}\right) \bmod p^{n}$.

From the following commutative diagram:

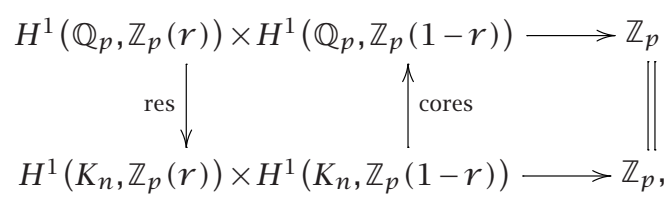

we have

$$
\begin{aligned}
\left(\xi, \int_{\mathbb{Z}_{p}^{\times}} x^{-r} \hat{\beta}\right) & =\left(\xi, \operatorname{cores}\left(\int_{1+p^{n} \mathbb{Z}_{p}} x^{-r} \hat{\beta}\right)\right)=\left(\operatorname{res}(\xi), \int_{1+p^{n} \mathbb{Z}_{p}} x^{-r} \hat{\beta}\right) \\
& \equiv\left(\xi_{n}, \int_{1+p^{n} \mathbb{Z}_{p}} x^{-r} \hat{\beta}\right) \equiv \xi_{n}\left(\beta_{n}\right)\left(\bmod p^{n}\right),
\end{aligned}
$$

this implies

$$
\left(\xi, \int_{\mathbb{Z}_{p}^{\times}} x^{-r} \hat{\beta}\right)=\lim _{\leftarrow} \xi_{n}\left(\beta_{n}\right)
$$

which is $\xi_{\infty}(\beta)$; this completes the proof.

THEOREM 7.4. The derivative $d \psi_{k} /\left.d k\right|_{k=1}=-\left(1-p^{-1}\right)^{-1} \gamma_{p}$.

Proof. This is equivalent to showing that $d \psi_{k}(\beta) /\left.d k\right|_{k=1}=-\left(1-p^{-1}\right)^{-1} \gamma_{p}(\beta)$, for all $\beta \in$ U.

Let $\mu=\mu_{a b} / \delta_{a b} \in \mathscr{D}_{1}\left(\mathbb{Z}_{p}, D\left(\mathbb{Q}_{p}(1)\right)\right)$ and $\mu^{\prime}=\left(-t x \mu_{\beta}\right) \in \mathscr{D}_{1}\left(\mathbb{Z}_{p}^{\times}, \mathbb{Q}_{p}\right)$, for all $\beta \in \mathcal{U}$. By Theorem 6.3 and Lemma 6.4(iii), we have

$$
\left[\mu, \mu^{\prime}\right]_{D\left(\mathbb{Q}_{p}(1)\right)}=-\left(\hat{p}, x^{-1} \hat{\beta}\right)_{\mathbb{Q}_{p}(1)}
$$

Taking the integral, we get

$$
-\int_{\mathbb{Z}_{p}^{\times}}\left[\mu, \mu^{\prime}\right]_{D\left(\mathbb{Q}_{p}(1)\right)}=\int_{\mathbb{Z}_{p}^{\times}}\left(\hat{p}, x^{-1} \hat{\beta}\right)_{\mathbb{Q}_{p}(1)} .
$$


By Lemmas 7.1 and 7.2, the left-hand side of (7.15) is

$$
\begin{aligned}
-\int_{\mathbb{Z}_{p}^{\times}}\left[\mu, \mu^{\prime}\right]_{D\left(\mathbb{Q}_{p}(1)\right)} & =-\int_{\mathbb{Z}_{p}^{\times}}\left[\frac{\mu_{a b}}{\delta_{a b}}, \mu^{\prime}\right] \\
& =-\int_{\mathbb{Z}_{p}^{\times}}\left[\mu_{a b}, \frac{\mu^{\prime}}{\delta_{a b}^{\vee}}\right] \\
& =-\int_{\mathbb{Z}_{p}^{\times}} \mu_{a b} \cdot \int_{\mathbb{Z}_{p}^{\times}} \frac{\mu^{\prime}}{\delta_{a b}^{\vee}} \\
& =-\left(1-\frac{1}{p}\right) \log \left(\frac{a}{b}\right) \cdot \frac{1}{\log \left(a^{-1} / b^{-1}\right)} \cdot \lim _{s \rightarrow 0} \frac{1}{s} \cdot \int_{\mathbb{Z}_{p}^{\times}}\langle x\rangle^{s}\left(-x \mu_{\beta}\right) \\
& =-\left(1-\frac{1}{p}\right) \lim _{s \rightarrow 0} \frac{1}{s} \cdot \int_{\mathbb{Z}_{p}^{\times}}\langle x\rangle^{s} \cdot x \mu_{\beta} \\
& =-\left.\left(1-\frac{1}{p}\right) \frac{d \psi_{k}}{d k}(\beta)\right|_{k=1} .
\end{aligned}
$$

By Lemma 6.4, the right-hand side of (7.15) is

$$
\begin{aligned}
\int_{\mathbb{Z}_{p}^{\times}}\left(\hat{p}, x^{-1} \hat{\beta}\right) & =\left(\int_{\mathbb{Z}_{p}^{\times}} \hat{p}, \int_{\mathbb{Z}_{p}^{\times}} x^{-1} \hat{\beta}\right)_{\mathbb{Q}_{p}(1)} \\
& =\left(\gamma_{p}, \kappa_{1}(\beta)\right)_{\mathbb{Q}_{p}(1)} \\
& =\gamma_{p}(\beta),
\end{aligned}
$$

hence we have the formula

$$
\left.\frac{d \psi_{k}}{d k}\right|_{k=1}=-\left(1-\frac{1}{p}\right)^{-1} \gamma_{p} .
$$

This completes the proof of the theorem.

ACKNOWLEDGMENT. The author would like to thank Professor Glenn Stevens deeply for his advice and help. The proofs of some lemmas in this paper are essentially from him, and the question in this paper was proposed by him.

\section{REFERENCES}

[1] Y. Amice and J. Vélu, Distributions p-adiques associées aux séries de Hecke, Journées Arithmétiques de Bordeaux (Conf., Univ. Bordeaux, Bordeaux, 1974), Astérisque, no. 2425, Soc. Math. France, Paris, 1975, pp. 119-131.

[2] P. Colmez, Théorie d'Iwasawa des représentations de de Rham d'un corps local [Iwasawa theory of de Rham representations of a local field], Ann. of Math. (2) 148 (1998), no. 2, 485-571 (French).

[3] E. de Shalit, Iwasawa Theory of Elliptic Curves with Complex Multiplication, Perspectives in Mathematics, vol. 3, Academic Press, Massachusetts, 1987.

[4] B. Ferrero and R. Greenberg, On the behavior of $p$-adic L-functions at $s=0$, Invent. Math. 50 (1978), no. 1, 91-102.

[5] J.-M. Fontaine, Le corps des périodes p-adiques [The field of p-adic periods], Astérisque (1994), no. 223, 59-111 (French). 
[6] R. Greenberg, Trivial zeros of p-adic L-functions, $p$-Adic Monodromy and the Birch and Swinnerton-Dyer Conjecture (Boston, MA, 1991) (B. Mazur and G. Stevens, eds.), Contemp. Math., vol. 165, Amer. Math. Soc., Rhode Island, 1994, pp. 149-174.

[7] R. Greenberg and G. Stevens, $p$-adic L-functions and p-adic periods of modular forms, Invent. Math. 111 (1993), no. 2, 407-447.

[8] B. Mazur, J. Tate, and J. Teitelbaum, On p-adic analogues of the conjectures of Birch and Swinnerton-Dyer, Invent. Math. 84 (1986), no. 1, 1-48.

[9] B. Perrin-Riou, Théorie d'Iwasawa des représentations p-adiques sur un corps local [Iwasawa theory of p-adic representations over a local field], Invent. Math. 115 (1994), no. 1, 81-161 (French).

[10] J.-P. Serre, Local Fields, Graduate Texts in Mathematics, vol. 67, Springer-Verlag, New York, 1979.

[11] S. Zhang, On explicit reciprocity law over formal group, to appear in Int. J. Math. Math. Sci.

[12]_ On exponential map, Thesis, Boston University, Massachusetts, 2000.

Shaowei Zhang: Department of Mathematics and Statistics, Boston University, MA 02215, USA

E-mail address: swz@bu.edu 


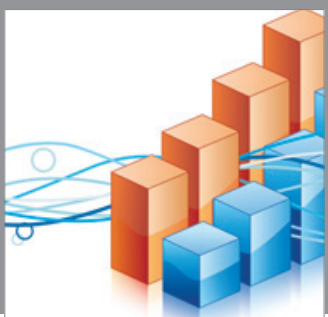

Advances in

Operations Research

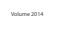

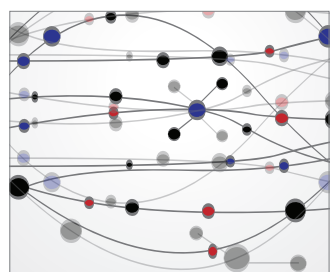

\section{The Scientific} World Journal
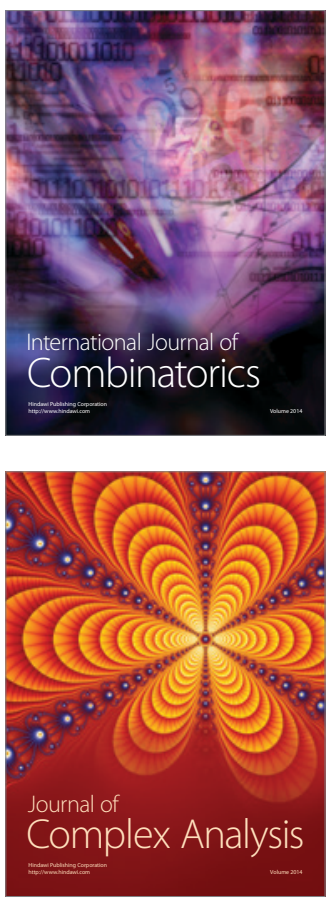

International Journal of

Mathematics and

Mathematical

Sciences
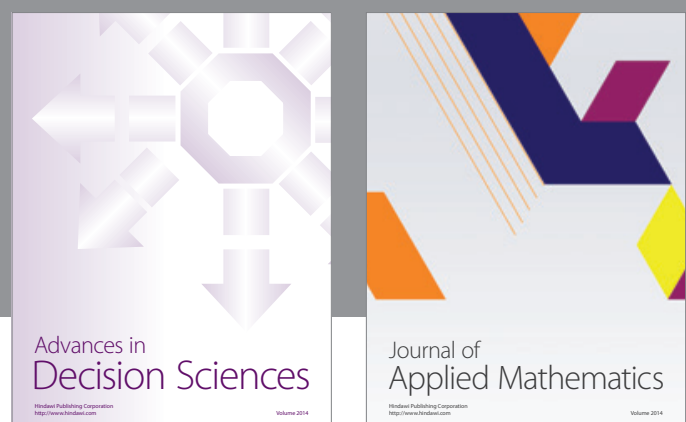

Journal of

Applied Mathematics
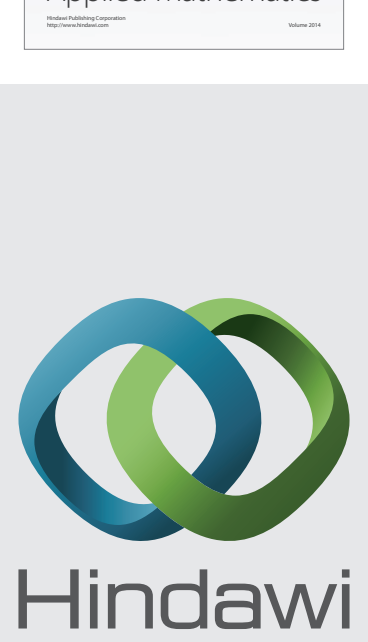

Submit your manuscripts at http://www.hindawi.com
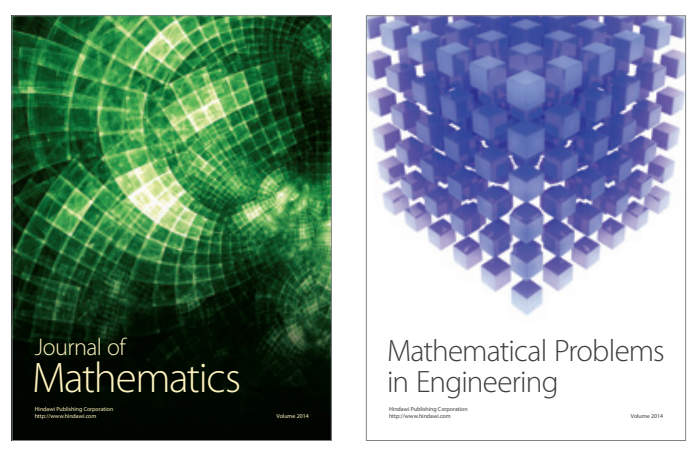

Mathematical Problems in Engineering
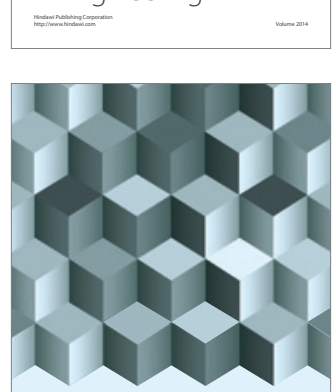

Journal of

Function Spaces
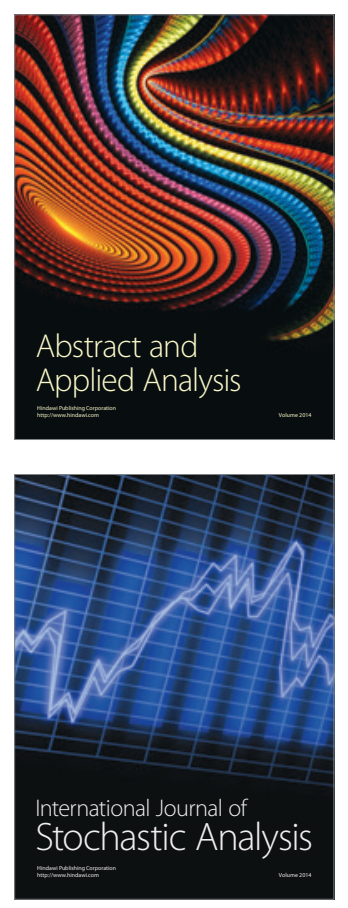

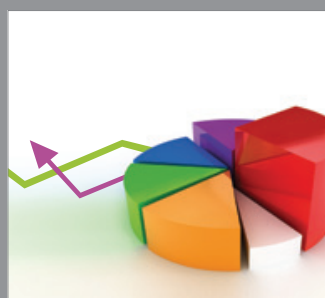

ournal of

Probability and Statistics

Promensencen
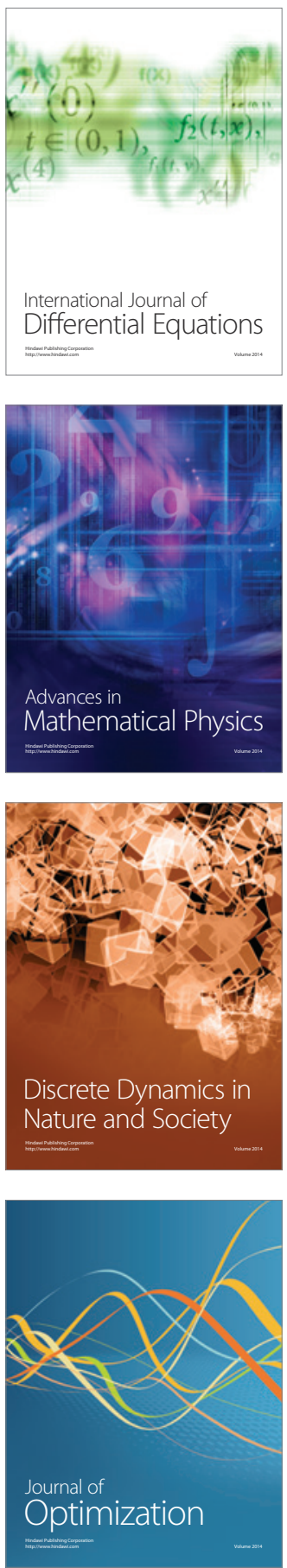